\title{
The Origin of the Color Charge into Quarks
}

\author{
Giovanni Guido \\ Department of Physics and Mathematics, High Scholl “C. Cavalleri” Parabiago, Milano, Italy \\ Email: gioguido54@gmail.com
}

How to cite this paper: Guido, G. (2019) The Origin of the Color Charge into Quarks. Journal of High Energy Physics, Gravitation and Cosmology, 5, 1-34. https://doi.org/10.4236/jhepgc.2019.51001

Received: June 25, 2018

Accepted: November 13, 2018

Published: November 16, 2018

Copyright $\odot 2019$ by author and Scientific Research Publishing Inc. This work is licensed under the Creative Commons Attribution International License (CC BY 4.0).

http://creativecommons.org/licenses/by/4.0/

\begin{abstract}
Showing the origin of the mass in an additional coupling between field quantum oscillators, we formulate a hypothesis of a geometrical structure of the oscillators of "fields-particles". In this way, we define the possible structure of quarks and hadrons (as the proton). This hypothesis is reasonable if one admits field oscillators composed by sub-oscillators at semi-quantum (IQuO) and in which a degree of internal freedom is definable. Using the IQuO model, we find the origin of the sign of electric charge in to particles and, in quarks, the isospin, the strangeness and colour charge. Finally, we formulate the structure of the gluons and the variation modality of the colour charge in quarks.
\end{abstract}

\section{Keywords}

Massive Coupling, "Aureus" Ratio, Geometrical Structure, IQuO, Semi-Quantum, Sub-Oscillator, Entangled Quantum Vacuum, Electric Charge, Isospin, Strangeness, Color, Gluon

\section{Introduction}

If we associate a wave behaviour to particles, you see the diffraction with electrons and photons, one can conjecture that "something" oscillates "inside" a particle. This conjecture is plausible even if the positivist literature has set aside it, because this is not directly verifiable with experiments. Instead, by this hypothesis, we could talk about internal "clock" with proper frequency $\left(\omega_{0}\right)$. On this basis, we could say that "the time is inside the particles". In the same way, then we could discuss about Compton wavelength $\left(\lambda_{c}\right)$ or the spin of the particles, as something of "space" "inside" these. All that pushes us to affirm that "the Space-Time is inside the particles". Since we always speak of particles being in space-time (ST), you see the conception of frame reference, thus we can say that particles and Space-Time define reciprocally themselves and if particles are also 
fields we can talk about Space-Time Field. From these ideas we can assume the permissible question of physically detect, even indirectly, the "internal" oscillation. We, previously, have given a positive response to this question stating [1] [2] that the sign of electric charge is related to the phase rotation of an "intrinsic oscillation", as well as the mass of the particles which is related [3] to "proper frequency" of this internal oscillation.

Thus, to talk about "internal" oscillation or Space-Time (ST) "inside" particles, it is the same as talking about "internal structure" of a particle. However, if we assume (you see the relativity) that elementary particles (quarks and leptons) are simply points in ST, while in quantum theory are fields $(\Psi)$, then we cannot consider them as composed by sub-particles, such as into nuclei or nucleons. However, if we want admit that inside particles there is an internal structure of "oscillators" and at the same time we want to be coherent with the relativity and Quantum Mechanics, then there is an only possible assertion: the idea of a structure of "coupled oscillators" which makes a particle as a unique and "not separable" object (see "elementary particle"). If, after, we recall that in quantum mechanics $(\mathrm{QM})$ theory the fields are represented as a set of coupled quantum oscillators, then we can give "particles" of the field an internal structure of coupled oscillators: this is the "hypothesis of structure" [1]. In this way we can think of quarks as geometric structures of coupled oscillators. However, speaking of structures of coupled oscillators does not imply that those structures are "rigid": the same we could talk about components in movement (see the proton spin and of the quarks which compose them). Nevertheless, the "hypothesis of structure" is based on a previously hypothesis of quantum oscillators at semi-quanta called IQuO (acronym of Intrinsic Quantum Oscillator), you see ref. [1] [2]. In truth, we already discussed an internal geometric structure of the elementary particles and, exactly, we talked [3] [4] about quarks ( $u, d, s)$ and (c, $b, t)$ as physical systems having a well-defined spatial geometric structure of coupled oscillators. Instead, in this paper we prefer to ignore previous works developing these structures; then we will explore the road that starts from the hypothesis of structure and leads us to formulate a hypothesis of quantum oscillators at semi-quanta called IQuO. We will then show the fundamental properties of the "IQuOs" and, through them, unveiling the meaning of fundamental physical properties [5], such as the mass of the particles, the sign of electric charge, the Y hypercharge and the isospin in the quark [6]. Besides, after processing the quarks with the IQuO model (quantum oscillators defined with semi-quanta and sub-oscillators), we define the structure of the "gluons" [6] and we find the origin of the "colour charge" and its structure in this model.

\section{Hypothesis of Structure}

\subsection{Massive Coupling}

If "the time is inside the particles" then any massive object can be seen as in "movement in time". We recall [3] the imaginary velocity in Space-Time 4-dim. 
which is given by the constant (c) in $\left[p_{4}=m c\right]$, (you see the relativity) and even the concept of energy a rest. So we can speak of mass-energy like the energy of movement in time: all this is coherent to relativity. This uniform motion in time recalls once again the "clock" that exists inside every particle-object as a periodic "motion". With proper frequency $\left(\omega_{0}\right)$, which corresponds to $\left[\omega_{0} \Leftrightarrow \tau\right]$, where $(\tau)$ is the proper time. The proper characteristic of a massive particle, associated with the proper time $(\tau)$ of an object, coincides with the proper mass $\left(m_{0}\right)$ of that object. Then, discussing the mass or mass-energy of a particle, it is the same that to consider the time of the clock which is inside them $\left[\omega_{o} \Leftrightarrow \tau \Leftrightarrow m_{o}\right.$. Then, based on QM [1]:

$$
\left\{\begin{array}{l}
E_{0}=m c^{2} \\
E_{0}=\hbar \omega_{0}
\end{array}\right\} \Rightarrow\left\{m=\frac{\hbar \omega_{0}}{c^{2}}\right\}
$$

If the frequency $\omega_{0}$ generates the proper time $\tau$ of a massive particle $[\tau=$ $\left.\hbar / m c^{2}\right]$, then for symmetry, there exists a wavelength $\lambda_{c}$ that originates the "proper space" of the particle [1]. Following De Broglie, we have:

$$
\left\{\begin{array}{l}
p_{0}=m c \\
p_{0}=\hbar \frac{2 \pi}{\lambda_{0}} \Rightarrow \lambda_{0}=\frac{\hbar}{m c} \equiv \lambda_{c}
\end{array}\right.
$$

We assert that $\left[\lambda_{c}=\hbar / m c\right]$ (i.e., the Compton wavelength) defines the spatial step of the proper ST of the massive particle. Now, combining the equation of the relativistic energy with the equations of De Broglie and Einstein, we have:

$$
\left\{E^{2}=m^{2} c^{4}+p^{2} c^{2} \Leftrightarrow \omega^{2}=\omega_{0}^{2}+k^{2} c^{2}\right\}
$$

The second equation is the dispersion relationship of waves, as described by the Klein-Gordon equation:

$$
\left\{\frac{\partial^{2} \Psi(x, t)}{\partial t^{2}}=c^{2} \frac{\partial^{2} \Psi(x, t)}{\partial x^{2}}-\omega_{0}^{2} \Psi(x, t)\right\}
$$

As is well known, this equation describes the oscillations in a set of pendulums coupled through springs [3] and scalar fields associated with massive particles with zero spin:

$$
\left\{\frac{\partial^{2} \Psi(x, t)}{\partial x^{2}}-\frac{\partial^{2} \Psi(x, t)}{c^{2} \partial t^{2}}=\left(\frac{m c}{\hbar}\right)^{2} \Psi(x, t)\right\} \Leftrightarrow\left\{\nabla^{2} \Psi(x, t)=\left(\frac{m c}{\hbar}\right)^{2} \Psi(x, t)\right\}
$$

We conjectured [3] that mass is a physical expression of the proper frequency $\left(\omega_{o}\right)$ related to a particular elastic coupling, which is in addition to the one already existing between the oscillators of the massless scalar field $(\Xi)$. This "additional coupling", which produces the mass in a scalar field $(\Xi)$, has been referred to as a "massive coupling" [7]. Then, we conjectured that the massive particle-field $(\underline{\underline{\Xi}})$ is originated by a "transversal coupling" $\left(\mathrm{T}_{0}\right)$ between the chains of oscillators of the scalar base field $(\boldsymbol{\Xi})$. All that can be represented in way figurative as shown in Figure 1. 


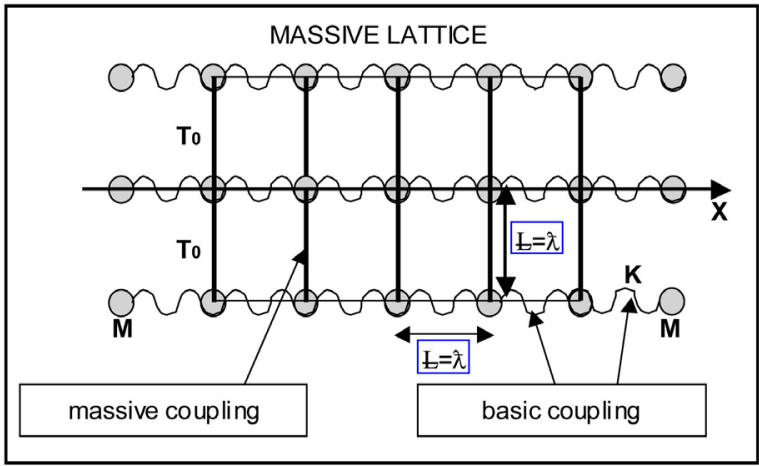

Figure 1. The massive field as a lattice of "pendulums" with springs.

Therefore, a massive particle can be represented by lattice with transversal coupling on an $\Xi$-scalar field. The massive particles are so constructed by the massive additional coupling which translates the "internal structure" of "coupled oscillators" into a geometric form.

\subsection{Hypothesis of Structure}

The massive particles are so originated by means of the massive additional coupling which builds the "internal structure" of "coupled oscillators" in geometric form.

A first geometric form we can find into proton considering an apparent coincidence between the Compton's wavelength of Planck's particle and the one of the proton. Following this coincidence and the "Hypothesis of Structure" [2], one can describe a proton as having a pentagonal geometric structure in which the constituent three quarks are coincident with three internal triangles (see the following Figure 2):

The vertices $(A, B, C)$ are coincident with the three centres of diffusion in the interaction electron-proton. The structure is so because one high lights an "aurea" (golden) relation between the proton and Plank's particle. Recall (see Figure 3) the "aureus" (golden) segments:

By property of the "golden segment" one has:

$$
\left\{\begin{array}{l}
\lambda_{\beta}=\left(\frac{\lambda_{\gamma}}{\phi}\right) \\
\lambda_{\gamma}=\phi^{2} \lambda_{\alpha}
\end{array}\right.
$$

where $(\phi)$ is the "aureus" number. If we denote now with $\boldsymbol{n}_{(p l, p)}$ the experimental numerical ratio between the Compton's wave length $\left(\lambda_{p l}\right)$ and $\left(\lambda_{p}\right)$, experimentally it's:

$$
\left(n_{(p l, p)}\right)=\left(\frac{\lambda_{p}}{\lambda_{p l}}\right)=\left(\frac{m_{p l}}{m_{P}}\right)=\left[\frac{(2.176450)(10)^{-8}}{(1.672623)(10)^{-27}}\right]=(1.301220)(10)^{19}
$$

the power $(10)^{(\mathrm{p})}$ can be a representative scale factor (s). Remember that is:

$$
\phi^{2}=(1.618034)^{2}=(2.618034)
$$




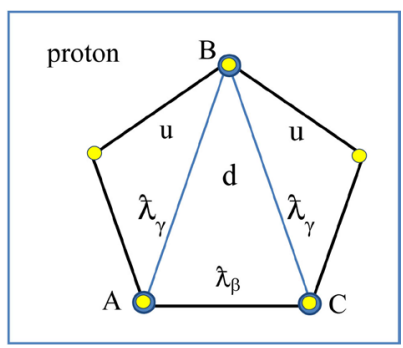

Figure 2. The geometric structure at quark of the proton.

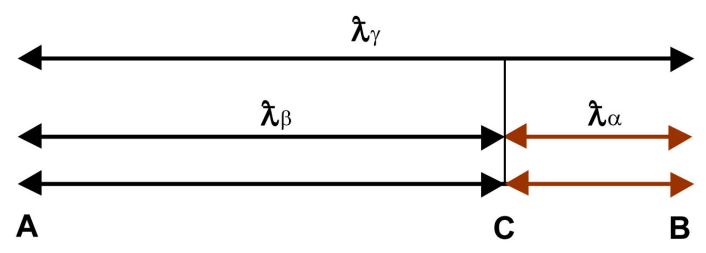

Figure 3. "Aureus segments".

We note that $\left[n_{(p l, p)} /(10)^{19}\right] \sim\left[(\phi)^{2} / 2\right]$ and $\left[2 n_{(p l, p)}=(\phi)^{2} k\right]$.

Then by Equation (2.6), we note there is an "aurea" relation, less than a factor $(10)^{p}$ between the proton and the Planck particle. In universe where the space-time is in expansion the spatial relations between some geometric structures could "dilate" increasing in scale. So we assume that a particular spatial relation between particles could be invariant to any scale. Therefore, we could state that a proton is an "aurea" particle because it follows the relation (2.6a); in this way, we can consider the three quark which compose it as three "aureae" triangles [(u), (u), (d)]. The same, using the Hypothesis of Structure, a quark can be represented by three elastically coupled quantum oscillators. This hypothesis could allow us to represent [1] the ( $u, d)$ quarks as structures of coupled quantum oscillators, you see the Figure 2. The representative structure [1] is similar to one of three "spheres" placed at the vertices of an aureus triangle and connected by "springs" ("junction oscillators"). Nevertheless, these structure can be possible (see [2] [3]) only if we use particular quantum oscillators defined "IQuO" (acronym of Intrinsic Quantum Oscillator). Besides, we need adding that the three quantum oscillators need to constitute an individual physical object, i.e. a quark, and this says us that we cannot separately detect the IQuO, as shown in Figure 4.

On these ideas, we discussed the "sub-structure" of quarks, which is applicable only to "particular" quantum oscillators (IQuO). This "particularity" is underlined in the necessity that the vertex-oscillator to connect to the other vertices trough junction oscillators has to be able to have in turn a structure of "hooks". All that induces us to talk about a "sub-structure" into quantum oscillator: we so need having an oscillator with more components. An oscillator of this type is highlighted only into quantum oscillator coupled to other oscillators. In fact, in the oscillation theory two coupled oscillators influence each other as if they are two "driven" oscillators. Just in a "classic" driven oscillator is highlighted a 


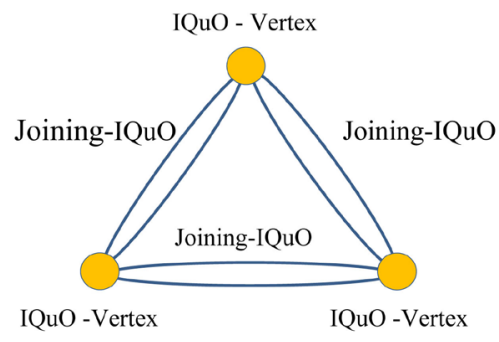

Figure 4. Quark sub-structure.

"structure" of components such as the "elastic" component and "absorptive" [3]. Therefore, this quantum oscillator with two components allows us to talk about a more elementary structure of it.

Another indication of a "composite structure" in quantum oscillator derives from its wave function (see Figure 5):

In fact, the quantum oscillator (with $n$ the quantum number and $n=1$ ) shows a wavefunction $(\Psi)$ with a pair of peaks in the probability of detecting the energy quanta of the oscillation: we can describe therefore the quantum oscillator with two sub-units of oscillation or "sub-oscillators". Into quantum oscillator with $(n=2)$ there are three peaks in wave function $(\Psi)$ which denote three sub-oscillators (see the Figure 5).

Not only, but two components in an oscillator encourage us to believe that the energy of the "quanta" is distributed between the two oscillating components.

We think the presence of two components in an oscillator causes the splitting of its quanta of energy into two energetic components in each sub-oscillators: this introduces the idea of "semi-quanta" (or individually "semi-quantum"). A quantum oscillator with a sub-structure made of sub-units of oscillation, or "sub-oscillators" and "semi-quanta" is called "IQuO" [2].

\section{The IQu0}

\subsection{The Quantum Oscillator at "Semi-Quanta"}

We will explain the origin of the IQuO concept. The elastic couplings of field quantum oscillators transform each quantum field oscillator in a coupled oscillator. Therefore, each field oscillator acts as a "driven" oscillator, which, following oscillations theory, is described by two components: the absorptive amplitude (or "inertial" amplitude) and the elastic amplitude. By using ([2] [8]) operators in phase plain $(q, p)$ we derive the following equation:

$$
\hat{q}(t)=\hat{q}_{e l}(0) \cos (\omega t)+\hat{q}_{a b s}(0) \sin (\omega t)
$$

Thus, [2] [3] [8] we can represent an oscillator as described in Figure 6.

Therefore, in a driven oscillator two components are highlighted: the "elastic" and "absorptive" components [3] [5] [8]. It is important to note that any type of driven-damped oscillator as well as the ones elastically coupled-to-other-oscillators, show a solution of the motion equations comprising of two oscillating components. These components are defined as "elastic" and "inertial" (or absorptive). 


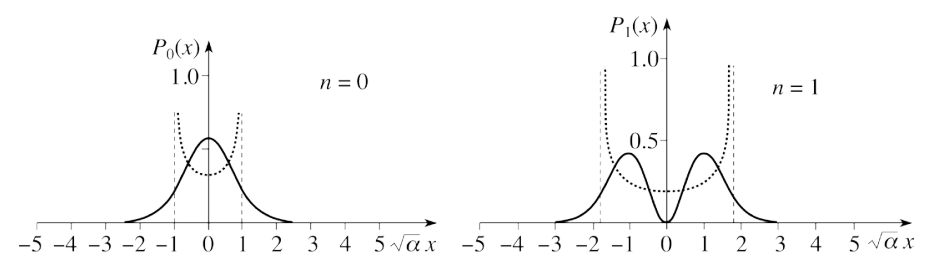

$$
\text { (a) }
$$

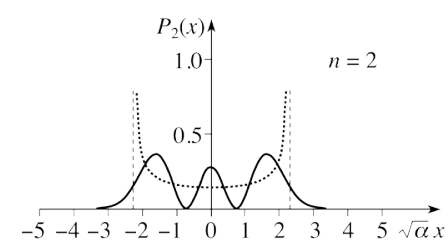

(c) (b)

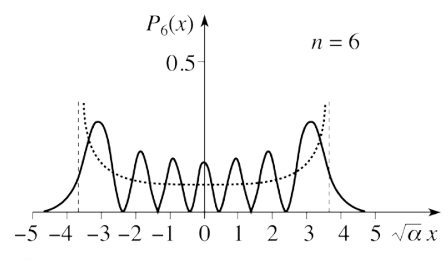

(d)

Figure 5. Probability function of quantum oscillator.

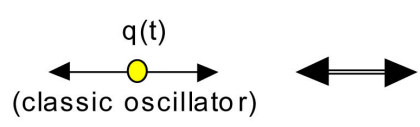

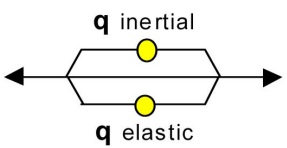

(Field's oscillator)

Figure 6. Oscillator at two-components.

This hypothesis of field quantum oscillators may seem "extreme"; however, we observe that in the electromagnetic interactions a photon "forces" an electron: at the point $(\mathrm{P})$ of interaction the individual quantum oscillator of the electronic field is driven by the individual quantum oscillator of the electromagnetic field (photon). In this case the electronic field oscillator is described by two components. The same for the photon or electromagnetic field. All that leads us to build a two-component representation of the quantum oscillators of both interacting fields and free fields, when the latter represent particles which are structured by coupled oscillators (see i.e. the quark). Consequently, the two operators of oscillators annihilation $(\boldsymbol{a})$ and creation $\left(\boldsymbol{a}^{+}\right)$which describe particles have two components, an elastic component and the other inertial:

$$
\left[a \rightarrow\left(a_{e l}, a_{i n}\right) ; a^{+} \rightarrow\left(a_{e l}^{+}, a_{i n}^{+}\right)\right]
$$

In this way, the quantum oscillator with two components could have a "2-dim. representation". This description cannot simply be a mere representation because the existence of two components (el, in) in quantum operators ( $a$, $\mathrm{a}^{+}$) per se implies a 2-dimensional aspect. This concept introduces an internal degree of freedom in the quantum oscillator. We could even say that this degree of freedom is highlighted in the interactions between particles (field couplings): see the electric charge.

Only this quantum oscillator with two components allows us to talk about a more elementary structure of it. The quantum oscillator of the field no longer will be described by the pair of operators $\left(a, a^{+}\right)$but by two pairs of operators $\left[\left(a_{e l}, a_{e l}^{+}\right),\left(a_{i n}, a_{i n}^{+}\right)\right]$where the absorptive component becomes the inertial component 


$$
\left\{\begin{array}{c}
\hat{a}_{t}=\hat{a}(t)_{\text {elastic }}+\hat{a}(t)_{\text {inertial }} \\
\hat{a}_{t}^{+}=\hat{a}^{+}(t)_{\text {elastic }}+\hat{a}^{+}(t)_{\text {inertial }}
\end{array}\right\} \Leftrightarrow\left\{\begin{array}{c}
\hat{a}_{t}=\hat{a}_{(e l)} \mathrm{e}^{-i \omega t}+\hat{a}_{(i n)} \mathrm{e}^{-i(\omega t-\pi / 2)} \\
\hat{a}_{t}^{+}=\hat{a}_{(e l)}^{+} \mathrm{e}^{i \omega t}+\hat{a}_{(i n)}^{+} \mathrm{e}^{i(\omega t-\pi / 2)}
\end{array}\right\}
$$

This double structure of the operators $\left(a, a^{+}\right)$splits the energy quanta of the quantum oscillator, giving:

$$
\begin{aligned}
{\left[H_{(n)}\right] } & =\left[U_{(n)}+K_{(n)}\right]=\left[\left(U_{(n)}\right)_{e l}+\left(K_{(n)}\right)\right] \\
& =\left[(2 n+1)\left(\frac{1}{4} \hbar \omega\right)_{e l}+(2 n+1)\left(\frac{1}{4} \hbar \omega\right)_{i n}\right]
\end{aligned}
$$

A structure with two sub-oscillators involves that when the energy "quantum" is in one of two sub-oscillator the other sub-oscillator cannot be empty of energy but having the value of $(\varepsilon=1 / 2 h v)$ defined "semi-quantum" (remember $\left[\varepsilon_{(n=1)}=\right.$ $(1+1 / 2) h v)])$. A structure with two components (elastic and "absorbing" or inertial) and two sub-oscillators would say that the "quantum" is composed by two $(\varepsilon=1 / 2 h v)$ semi-quantum. Then we conjecture energy values of $[(\varepsilon=$ $1 / 4 h v)]$, indicated as "empty semi-quantum" and symbol (o), and another [ $\varepsilon=$ $(1 / 4 h v)]$, indicated as "full semi-quantum" and symbol $(\bullet)$. We will obtain the probabilistic representation of the "semi-quantic" oscillator at a given instant (Figure 7).

An IQuO $\left.\left[\varepsilon_{n}=(n+1 / 2) h v\right)\right]$ will be represented by empty semi-quantum $(\varepsilon(\mathbf{o})=1 / 4 h v)$, and full semi-quantum $[\varepsilon(\bullet)=(1 / 2 h v)]$; so $\left[\varepsilon_{n}=(\mathrm{n}+1 / 2) h v\right)=$ $(n(1 / 2+1 / 2)+(1 / 4+1 / 4)) h v]$. We note that the semi-quantum flowing from a sub-oscillator to other.

We can represent the annihilation operator (a), and the creation operator $\left(a^{+}\right)$ trough new operators built on the full semi-quantum $(\bullet)$ and empty semi-quantum (o):

$$
\left\{\begin{array}{c}
\left\{\left(\begin{array}{cc}
\hat{a}_{e l} \equiv(\hat{\bullet})_{e l} & \hat{a}_{i n} \equiv(\hat{\mathrm{o}})_{i n} \\
\hat{a}_{e l}^{+} \equiv\left(\hat{\mathrm{o}}^{+}\right)_{e l} & \hat{a}_{i n}^{+} \equiv\left(\hat{\bullet}^{+}\right)_{i n}
\end{array}\right)\right\} \Leftrightarrow\left\{\left(\begin{array}{cc}
\hat{a}_{e l} \equiv(\hat{\mathrm{o}})_{e l} & \hat{a}_{i n} \equiv(\hat{\bullet})_{i n} \\
\hat{a}_{e l}^{+} \equiv\left(\hat{\bullet}^{+}\right)_{e l} & \hat{a}_{i n}^{+} \equiv\left(\hat{\mathrm{o}}^{+}\right)_{i n}
\end{array}\right)\right\} \\
\mathrm{B}_{1} \text {-Matrix } \\
\mathrm{B}_{2} \text {-Matrix }
\end{array}\right.
$$

The "commutation relation" of theoperators $((\bullet),(\mathbf{o}))$ have been reported in [2] and [3].

Here, there is a synthesis:

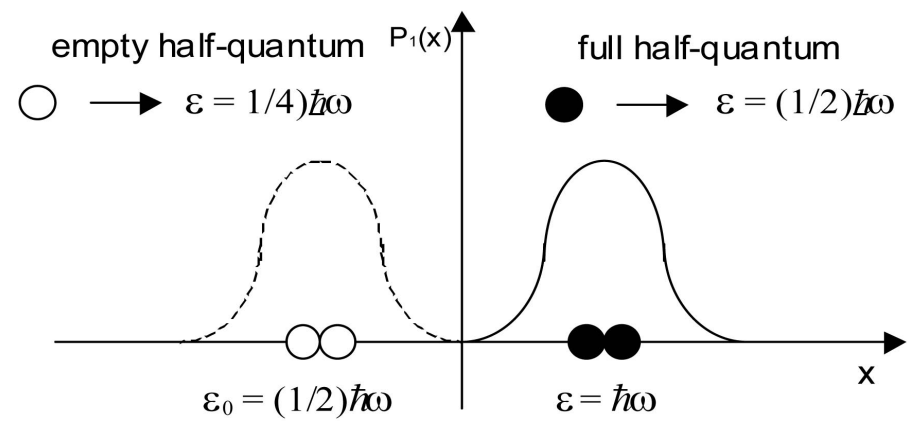

Figure 7. The probabilistic representation of the semi-quantum oscillator. 


$$
\left\{\begin{array}{ll}
{\left[\bullet_{e l}^{+}, \mathrm{o}_{e l}\right]=0 ;} & {\left[\bullet_{i n}^{+}, \mathrm{o}_{i n}\right]=0} \\
{\left[\mathrm{o}_{e l}^{+}, \bullet_{e l}\right]=0 ;} & {\left[\mathrm{o}_{i n}^{+}, \bullet_{i n}\right]=0} \\
{\left[\bullet_{e l}^{+}, \mathrm{o}_{i n}\right]=\frac{i}{2} ;} & {\left[\mathrm{o}_{e l}^{+}, \bullet_{i n}\right]=\frac{i}{2}} \\
{\left[\bullet_{i n}^{+}, \mathrm{o}_{e l}\right]=\frac{i}{2} ;} & {\left[\mathrm{o}_{i n}^{+}, \bullet_{e l}\right]=\frac{i}{2}}
\end{array}\right\}
$$

Then the Equation (3.1) in IQuO-representation will be:

$$
\begin{aligned}
& {[\Psi(t)] \equiv\left(\begin{array}{l}
\hat{a}_{r^{\prime}}^{+}(t) \\
\hat{a}_{r^{\prime}}(t)
\end{array}\right)=\left\{\begin{array}{l}
\hat{a}_{r^{\prime}}^{+}(t)=\left(\hat{\bullet}^{+}\right)_{e l}\left[\exp \left(i r^{\prime} \omega t\right)\right]+\left(\hat{\mathrm{o}}^{+}\right)_{i n}\left[\exp \left(i\left(r^{\prime} \omega t-\pi / 2\right)\right)\right] \\
\hat{a}_{r^{\prime}}(t)=(\hat{\mathrm{o}})_{e l}\left[\exp \left(-i r^{\prime} \omega t\right)\right]+(\hat{\bullet})_{i n}\left[\exp \left(-i\left(r^{\prime} \omega t-\pi / 2\right)\right)\right]
\end{array}\right.} \\
& \text { with } \vec{\Psi}(t) \equiv\left[\vec{a}^{+}(t)+\vec{a}(t)\right]=\left[\left(a_{e l}^{+}(t)+a_{i n}^{+}(t)\right)+\left(a_{e l}(t)+a_{i n}(t)\right)\right]
\end{aligned}
$$

where $\left(r^{\prime}= \pm 1\right)$ is connected to the direction of phase rotation.

For $\left(r^{\prime}=+1\right)$ the two equivalent representative ( 2 rows $) \times(2$ columns $\left.)\right)$ matrices (see Equation (3.1)) are:

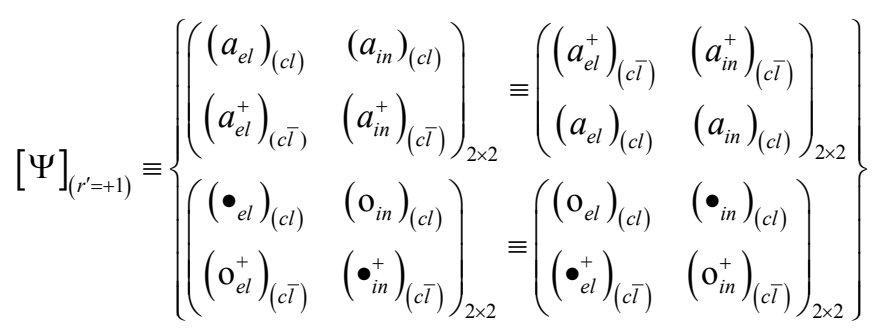

Omitting the time and the phase but highlighting the rotation direction (clockwise (cl)-anticlockwise ( $\underline{\mathbf{c l}})$ ). Besides, remember that inertial component is shifted to $(\pi / 2)$ by the elastic component. There are different configurations of $\mathrm{IQuO}$ in the pairs $((\bullet),(\mathbf{o}))$ but all equivalents, as:

$$
\left[\left(\bullet_{i n}^{+}, \bullet_{e l}^{+}\right),\left(\underline{\mathrm{o}}_{e l}, \underline{\mathrm{o}}_{i n}\right)\right] \Leftrightarrow\left[\left(\mathrm{o}_{i n}^{+}, \mathrm{o}_{e l}^{+}\right),\left(\underline{\bullet}_{e l}, \bullet_{i n}\right)\right]
$$

An IQuO (see Figure 8) can so have the following graphically representation into phase plain (q, ip), see [2] [3]:

Because two sub-oscillators compose the oscillator, going in the representation of wave function scalar $(\Psi(x(t))$ and highlighting the phase rotation, we will obtain a bi-dimensional representation of oscillators with $\left[\left(a_{e l}, a_{e l}^{+}\right),\left(a_{i n}, a_{i n}^{+}\right)\right]$. It will be graphically (Figure 9(a)) and into instant:

Note that the configuration is variable to the pass of time.

The next configurations (Figure 9(b)) can be the following:

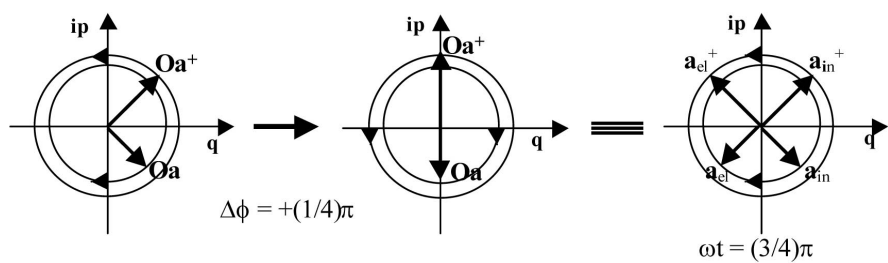

Figure 8. The two-components oscillator in phase plain. 


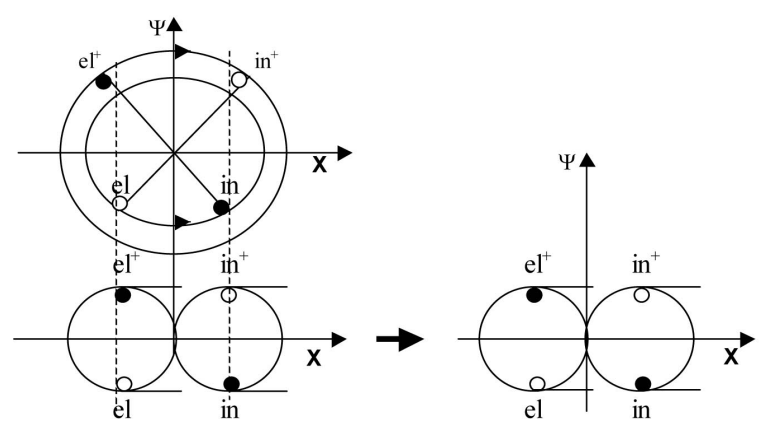

(a)
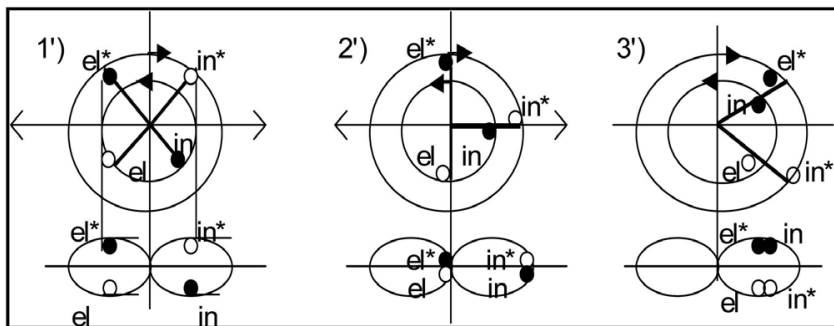

(b)

Figure 9. (a) Graphic representation of an IQuO; (b) Next two configurations.

The 8-configuration will coincide with 1-configuration.

In the matrix representation, omitting the time and phase, this configuration is:

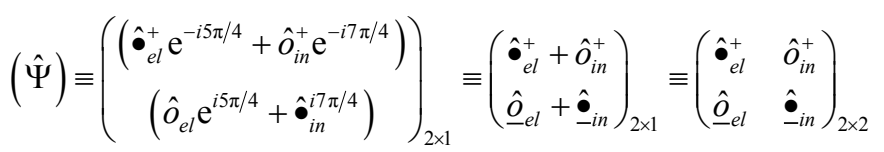

where $\left(\underline{\mathbf{o}}_{e b} \underline{\mathbf{o}}_{i n}\right)$ is the couple with anti-clockwise phase rotation, while $\left(\mathrm{o}_{i n}^{+}, \bullet_{e l}^{+}\right)$ is the couple with clockwise phase rotation. Here we represent a matrix $(2 \times 1)$ as matrix $(2 \times 2)$ of components of $\Psi$. Note the energy exchange between full semi-quanta $(\bullet)$ with empty semi-quanta (o) (represented by $[(\bullet) \Leftrightarrow(\mathbf{o})]$ ) happens in any point of axis $\mathrm{x}$ (see the equivalence between matrices in Equation (3.6). This because the operator $\left[\left(\bullet_{e l}^{+}\right)_{\rightarrow}\right]$ creates a semi-quantum $(\bullet)$ on the right, while $\left[\left(\mathrm{o}_{e l}\right)_{\rightarrow}\right]$ annihilates a semi-quantum (o) on the right: the same for $\left(\mathrm{o}_{i n}^{+}\right)_{\rightarrow}$ and $\left(\bullet_{i n}\right)_{\rightarrow}$. Therefore, the two applications are equivalents because they indicate the propagation of a semi-quantum $(\bullet)$ the right.

Two IQuO $\left(\Psi_{1}, \Psi_{2}\right)$ can be combined (Figure 10) in one zone of superposition $(x)$ :

Using the $(\oplus)$ combination operation [2]:

$$
\left(\Psi_{1} \oplus \Psi_{2}\right)=\left\{\hat{\Psi}_{1}[\hat{\Omega}] \hat{\Psi}_{2}\right\}
$$

where the right side of Equation (3.8) contains the two representative matrices of the $\Psi$-IQuO and the $\Omega$-operation defined as:

$$
\hat{\Omega}=\left(\overline{\mathrm{X}} \cdot \hat{\Sigma}_{(\bullet \bullet \circ)} \cdot \hat{\varphi}\right)=\cdot\left[(\overline{\mathrm{X}})(\bar{\Sigma}(\bullet \Leftrightarrow \mathrm{o}))\left(\begin{array}{cc}
\mathrm{e}^{-i \Delta \varphi} & 0 \\
0 & \mathrm{e}^{i \Delta \varphi}
\end{array}\right)_{2 \times 2}\right]
$$




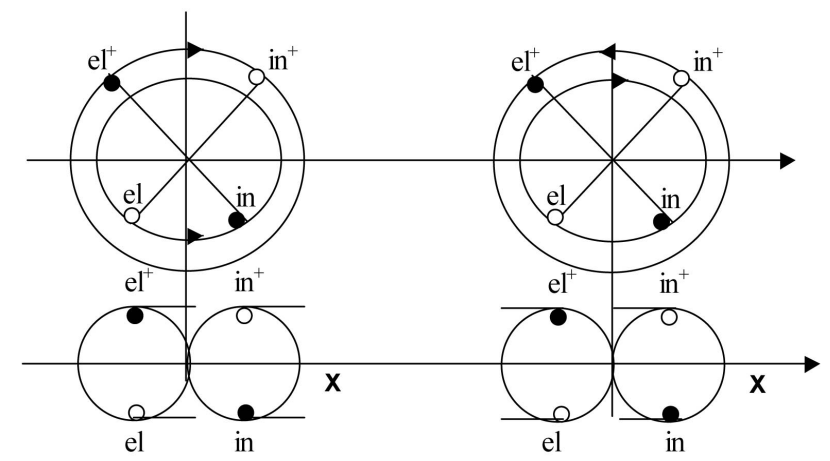

Figure 10. Two IQuO before of elastic coupling.

- $(\mathrm{X})$ is the operation representing the diagonal coupling of matrix elements

- $(\Sigma)$ is the operation representing the energy exchange between semi-quanta of different two IQuO, (exchange of full semi-quanta $(\bullet)$ of the first IQuO with empty semi-quanta (o) of the other: $[(\bullet) \Leftrightarrow(\mathbf{o})])$

- $(\varphi)$ denotes the reduction of the wave function $(\Psi)$ between phase shifts $(\Delta \varphi)$

$$
\begin{aligned}
& \hat{\varphi}=\left(\begin{array}{cc}
\mathrm{e}^{-i \Delta \varphi} & 0 \\
0 & \mathrm{e}^{i \Delta \varphi}
\end{array}\right) \\
& \text { if }(\Delta \varphi=0) \Rightarrow \hat{\varphi}_{(\Delta \varphi=0)}=\left(\begin{array}{ll}
1 & 0 \\
0 & 1
\end{array}\right)
\end{aligned}
$$

Then it is:

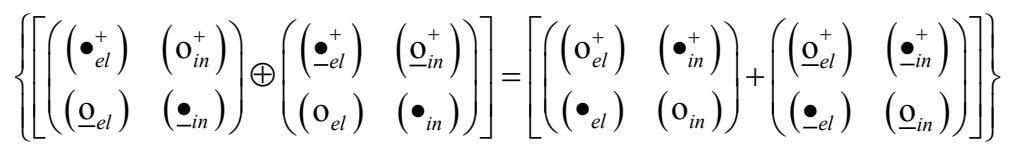

Note the X-operation is derived by associative property of addition (you see the Equation (3.5)):

$$
\begin{aligned}
\left(\Psi_{1} \oplus \Psi_{2}\right)= & \left\{\left[\left(\bullet_{e l 2}^{+}\right)+\left(\mathrm{o}_{i n 2}^{+}\right)\right]+\left[\left(\underline{\mathrm{o}}_{e l 2}\right)+\left(\boldsymbol{\bullet}_{i n 2}\right)\right]\right\} \\
& +\left\{\left[\left(\boldsymbol{\bullet}_{e l 1}^{+}\right)+\left(\underline{\mathrm{o}}_{i n 1}^{+}\right)\right]+\left[\left(\mathrm{o}_{e l 1}\right)+\left(\bullet_{i n 1}\right)\right]\right\} \\
= & \left\{\left[\left(\bullet_{e l 1}^{+}\right)+\left(\mathrm{o}_{i n 1}^{+}\right)\right]+\left[\left(\boldsymbol{\bullet}_{e l 2}\right)+\left(\mathrm{o}_{i n 2}\right)\right]\right\} \\
& +\left\{\left[\left(\underline{\mathrm{o}}_{e l 1}^{+}\right)+\left(\underline{\bullet}_{i n 1}^{+}\right)\right]+\left[\left(\boldsymbol{\bullet}_{e l 2}\right)+\left(\underline{\mathrm{o}}_{i n 2}\right)\right]\right\} \\
= & \left(\Phi_{\underline{c l}}+\Phi_{c l}\right)
\end{aligned}
$$

Graphically, the final result (Figure 11) is:

With

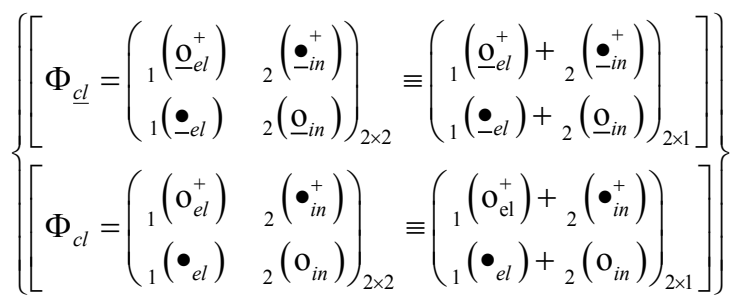




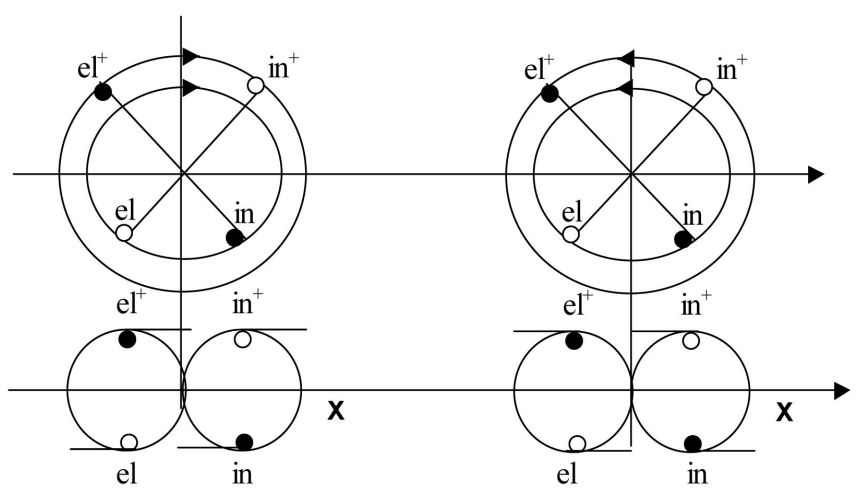

Figure 11. Two IQuO whit phase rotation unidirectional.

where the numbers on the left indicate the two sub-oscillators. Note that the ( $\Psi_{1}$ $\oplus \Psi_{2}$ ) coupling of two IQuO generates two particles-IQuO with opposite direction of the phase rotation $\left[\left(\Phi_{\text {anti-cl }}\right)+\left(\Phi_{\mathrm{cl}}\right)\right]$. Besides, note here the energy exchange $[(\bullet) \Leftrightarrow(\mathbf{o})]$ between $\left[\left(a_{e l}^{+} \Leftrightarrow a_{e l}\right),\left(a_{i n}^{+} \Leftrightarrow a_{i n}\right)\right]$ not happens in any point (see Figure 11): the $\Psi$-IQuO is so essentially different from $\Phi$-IQuO. We called $\Psi-\mathrm{IQuO}$ type as "Fermion" while $\Phi-\mathrm{IQuO}$ as "Boson". That is because we think that the $\mathrm{IQuO}$ are the fundamental quantum oscillators of all fields-particles.

\subsection{The Electric Charge}

An advantage in treating the field oscillators using the IQuO is that the "2-dimensional" representation (Figure 9) allows us" to distinguish the direction of rotation of the phase associated to oscillations having an only direction of phase rotation". In fact, taking into account both the distribution of semi-quanta inside the two sub-oscillators of an $\Phi-\mathrm{IQuO}\left(\mathrm{I}_{1}\right)$ and both their movement concerning the phase, another IQuO $\left(\mathrm{I}_{2}\right)$ in coupling with first IQuO $\left(I_{1}\right)$ it could detect the direction of rotation of the phase of $\left(\mathrm{I}_{1}\right)$.

Note that IQuO $\mathrm{I}_{2}$ ( $\Psi$-type) could be the representative quantum of a particle of interaction (i.e. photon). So, the direction of rotation of the phasedetermines a new degree of freedom in a quantum oscillator with semi-quanta. This degree of freedom admits two possibilities that would be interpreted as "the sign of the electric charge" of a particle. We conjecture that the IQuO $\left[\left(\Phi_{\text {anti-cl }}\right),\left(\Phi_{\mathrm{cl}}\right)\right]$ can represent particles with opposite sign of electric charge [2]. This important result marks a turning point in the understanding of the physics of the interactions. We recall that the electric charge is in correlation with the generator of gauge transformation $\mathrm{SU}(2)$ which Pauli's matrix is $\left(\sigma_{3}\right)$ [7] [8]; so, we can derive the $Q$ electric charge from the following equation, using the matrices $(\Phi)$ of the Equation (3.12):

$$
\left\{\begin{array}{l}
Q_{\underline{c l}}=\int\left[\left(\Phi_{\underline{c l}}^{+}\right) \hat{\sigma}_{3}\left(\Phi_{\underline{c l}}\right)\right] \mathrm{d} V=+1 \\
Q_{c l}=\int\left[\left(\Phi_{c l}^{+}\right) \hat{\sigma}_{3}\left(\Phi_{c l}\right)\right] \mathrm{d} V=-1
\end{array}\right\}
$$

Using the well-known definition of the electric charge [2] and the commuta- 
tion relations with semi-quanta (see Equation (3.4) Appendix in [2]) it then follows that:

$$
\begin{aligned}
& Q_{c l}=\int\left[\left(\left(\hat{\Phi}_{(-)}^{+}\right)\right) \hat{\sigma}_{3}\left(\left(\hat{\Phi}_{(-)}\right)\right)\right] \mathrm{d} V=\int\left(\begin{array}{c}
\left(\hat{\boldsymbol{\bullet}}_{e l}^{+} \mathrm{e}^{i \pi / 2}+\hat{\boldsymbol{\bullet}}_{i n}^{+}\right) \\
\left(\hat{o}_{e l} \mathrm{e}^{i \pi}+\hat{o}_{i n}^{i 3 \pi / 2}\right)
\end{array}\right)\left(\begin{array}{c}
\left(\hat{o}_{e l} \mathrm{e}^{-i \pi / 2}+\hat{o}_{i n}\right) \\
-\left(\hat{\boldsymbol{\bullet}}_{e l}^{+} \mathrm{e}^{-i \pi}+\hat{\boldsymbol{\bullet}}_{i n}^{+} \mathrm{e}^{-i 3 \pi / 2}\right)
\end{array}\right) \mathrm{d} V \\
& =\left[\left(\left(\hat{\boldsymbol{\bullet}}_{e l}^{+} \mathrm{e}^{i \pi / 2}\right)+\hat{\boldsymbol{\bullet}}_{i n}^{+}\right)\left(\hat{o}_{e l} \mathrm{e}^{-i \pi / 2}+\hat{o}_{i n}\right)-\left(\hat{o}_{e l} \mathrm{e}^{i \pi}+\hat{o}_{i n}^{i 3 \pi / 2}\right)\left(\hat{\boldsymbol{\bullet}}_{e l}^{+} \mathrm{e}^{-i \pi}+\hat{\boldsymbol{\bullet}}_{i n}^{+} \mathrm{e}^{-i 3 \pi / 2}\right)\right] \\
& =\left(i \hat{\bullet}_{e l}^{+} \hat{o}_{i n}-i \hat{\bullet}_{e l}^{+} \mathrm{e}^{-i \pi} \hat{o}_{i n}^{i 3 \pi / 2}\right)+\left(\hat{\bullet}_{i n}^{+} \hat{o}_{e l} \mathrm{e}^{-i \pi / 2}-\hat{\bullet}_{i n}^{+} \mathrm{e}^{-i 3 \pi / 2} \hat{o}_{e l} \mathrm{e}^{i \pi}\right) \\
& =\left[\hat{\bullet}_{e l}^{+}, \hat{o}_{e l}\right]+\left[\hat{\boldsymbol{\bullet}}_{i n}^{+}, \hat{o}_{i n}\right]+i\left[\hat{\boldsymbol{\bullet}}_{e l}^{+}, \hat{o}_{i n}\right]+i\left[\hat{o}_{e l}, \hat{\boldsymbol{\bullet}}_{i n}^{+}\right]=-1 \\
& Q_{\underline{c l}}=\int\left[\left(\left(\hat{\Psi}_{(+)}^{+}\right)\right) \hat{\sigma}_{3}\left(\left(\hat{\Psi}_{(+)}\right)\right)\right] \mathrm{d} V=\int\left(\begin{array}{c}
\left(\hat{o}_{e l} \mathrm{e}^{-i \pi}+\hat{o}_{i n} \mathrm{e}^{-i \pi / 2}\right) \\
\left(\hat{\boldsymbol{\bullet}}_{e l}^{+} \mathrm{e}^{i \pi / 2}+\hat{\boldsymbol{\bullet}}_{i n}^{+}\right)
\end{array}\right)\left(\begin{array}{c}
\left(\hat{\bullet}_{e l}^{+} \mathrm{e}^{i \pi}+\hat{\boldsymbol{\bullet}}_{i n}^{+} \mathrm{e}^{i \pi / 2}\right) \\
-\left(\hat{o}_{e l} \mathrm{e}^{-i \pi / 2}+\hat{o}_{i n}\right)
\end{array}\right) \mathrm{d} V \\
& =\left[\left(\hat{o}_{e l} \mathrm{e}^{-i \pi}+\hat{o}_{i n} \mathrm{e}^{-i \pi / 2}\right)\left(\hat{\boldsymbol{\bullet}}_{e l}^{+} \mathrm{e}^{i \pi}+\hat{\boldsymbol{\bullet}}_{i n}^{+} \mathrm{e}^{i \pi / 2}\right)-\left(\hat{\boldsymbol{\bullet}}_{e l}^{+} \mathrm{e}^{i \pi / 2}+\hat{\boldsymbol{\bullet}}_{i n}^{+}\right)\left(\hat{o}_{e l} \mathrm{e}^{-i \pi / 2}+\hat{o}_{i n}\right)\right] \\
& =\hat{o}_{e l} \hat{\bullet}_{e l}^{+}+\hat{o}_{e l} \hat{\bullet}_{i n}^{+} \mathrm{e}^{-i \pi / 2}+\hat{o}_{i n} \hat{\bullet}_{e l}^{+} \mathrm{e}^{i \pi / 2}+\hat{o}_{i n} \hat{\boldsymbol{\bullet}}_{i n}^{+} \\
& -\hat{\boldsymbol{\bullet}}_{e l}^{+} \hat{o}_{e l}-\hat{\boldsymbol{\bullet}}_{e l}^{+} \hat{o}_{i n} \mathrm{e}^{i \pi / 2}-\hat{\boldsymbol{\bullet}}_{i n}^{+} \hat{o}_{e l} \mathrm{e}^{-i \pi / 2}-\hat{\boldsymbol{\bullet}}_{i n}^{+} \hat{o}_{i n} \\
& =\left[\hat{o}_{e l}, \hat{\boldsymbol{\bullet}}_{e l}^{+}\right]+\left[\hat{o}_{i n}, \hat{\boldsymbol{\bullet}}_{i n}^{+}\right]+i\left[\hat{\boldsymbol{\bullet}}_{i n}^{+}, \hat{o}_{e l}\right]+i\left[\hat{o}_{i n}, \hat{\boldsymbol{\bullet}}_{e l}^{+}\right]=+1
\end{aligned}
$$

where $\left[\left(\Phi_{-}\right)=\left(\Phi_{\mathrm{cl}}\right),\left(\Phi_{+}\right)=\left(\Phi_{\mathrm{cl}}\right)\right]$ and $\sigma_{3}$ is a Pauli's matrix.

Note, besides, the conjugation operation transforms the semi-quantum operators:

$$
[(\bullet) \rightarrow(\mathrm{o}),(\mathrm{o}) \rightarrow(\bullet)]
$$

\subsection{The $\operatorname{IQuO}_{(n=0)}$}

The vacuum state of an "isolated" $\mathrm{IQuO}_{(n=0)}$ will be graphically represented (Figure 12):

Even here we can have some possible representations.

One of these (A-representation) can be the following (Figure 13):

With representative matrices $(2 \times 1)$ :

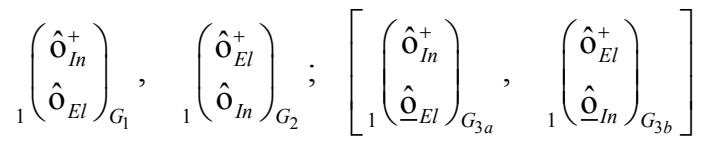

The conjugate representation (Figure 14) is:

And the conjugate matrices:

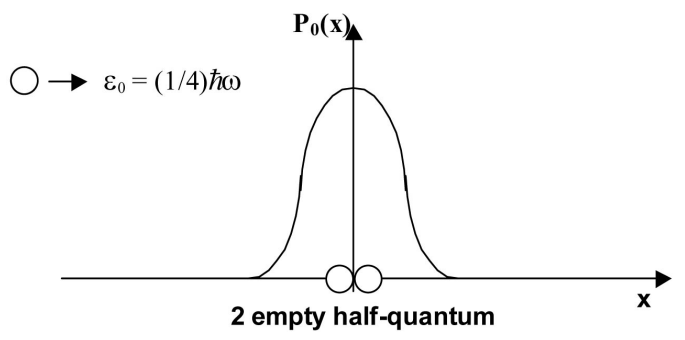

Figure 12. $\mathrm{IQuO}_{(\mathrm{n}=0)}$. 


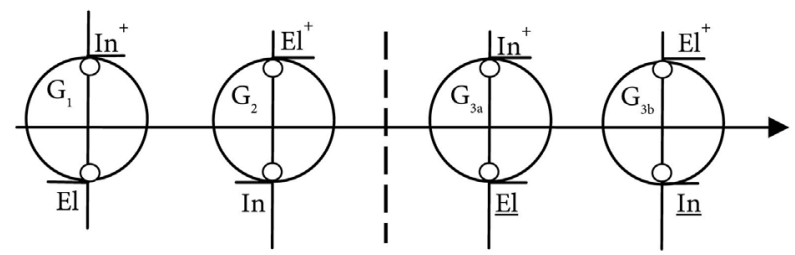

Figure 13. Possible A-representations of isolated sub-oscillator.

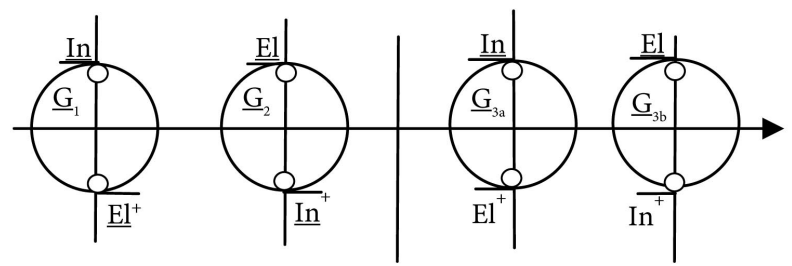

Figure 14. Conjugate A-representation of sub-oscillator.

$$
\left(\begin{array}{c}
\hat{\mathrm{o}}_{E l}^{+} \\
\underline{\hat{\mathrm{o}}}_{I n}
\end{array}\right)_{\underline{G}_{1}}, \quad\left(\begin{array}{l}
\hat{\mathrm{o}}_{I n}^{+} \\
\underline{\hat{\mathrm{o}}}_{E l}
\end{array}\right)_{\underline{G}_{2}} ;\left[\left(\begin{array}{c}
\hat{\mathrm{o}}_{E l}^{+} \\
\underline{\hat{\mathrm{o}}}_{I n}
\end{array}\right)_{\underline{G}_{3 a}},\left(\begin{array}{c}
\hat{\mathrm{o}}_{I n}^{+} \\
\underline{\hat{\mathrm{o}}}_{E l}
\end{array}\right)_{\underline{G}_{3 b}}\right]
$$

We note that the two possibilities $\left(G_{3 \mathrm{a}}, G_{3 \mathrm{~b}}\right)$ are indeed one only possibility because $\left(G_{3 \mathrm{a}}\right)^{+}=\left(G_{3 \mathrm{~b}}\right)$; then we admit one only possibility $\rightarrow\left(G_{3}\right)_{\mathrm{A}}$. We obtain: (Figure 15)

With representative matrices:

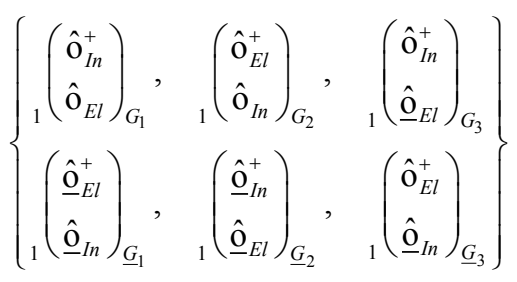

The other possible representation (B-representation) is: (Figure 16)

Which becomes: (Figure 17)

Nevertheless note $\left[\left(G_{1}\right)_{\mathrm{B}-\text { rep }}=\left(\underline{G}_{2}\right)_{\mathrm{A} \text {-rep }} ;\left(G_{2}\right)_{\mathrm{B} \text {-rep }}=\left(\underline{G}_{1}\right)_{\mathrm{A} \text {-rep }}\right]$; it follows that for $\left[\left(G_{1}\right),\left(G_{2}\right)\right]$ the two representation are coincident, instead for $\left(G_{3}\right)$ it's not possible: $\left[\left(G_{3}\right)_{\mathrm{A}-\text { rep }} \neq\left(G_{3}\right)_{\text {B-rep }}\right]$.

Two are the possibilities: the combinations $\left[G_{\mathrm{i} 3}, G_{\mathrm{i} 3}\right]_{\mathrm{B}-\text { rep }}$ have not physical meaning or we can admit a combination between $\left[\left(G_{3}\right)_{\mathrm{A}-\text { rep }},\left(G_{3}\right)_{\mathrm{B}-\text { rep }}\right]$. We opt for the second:

$$
\left\{\begin{array}{c}
\left(\begin{array}{c}
\hat{\mathrm{o}}_{I n}^{+} \\
\hat{\mathrm{o}}_{E l}
\end{array}\right)_{G_{3 A}} \oplus\left(\begin{array}{l}
\hat{\mathrm{o}}_{I n}^{+} \\
\hat{\mathrm{o}}_{E l}
\end{array}\right)_{G_{3 B}} \\
\left(\begin{array}{l}
\hat{\mathrm{o}}_{E l}^{+} \\
\hat{\mathrm{o}}_{I n}
\end{array}\right)_{\underline{G}_{3 A}} \oplus\left(\begin{array}{l}
\hat{\mathrm{o}}_{E l}^{+} \\
\underline{\mathrm{o}}_{I n}
\end{array}\right)_{\underline{G}_{3 B}}
\end{array}\right\} \equiv\left\{\begin{array}{l}
\left(\begin{array}{c}
\hat{\mathrm{o}}_{I n}^{+}+\hat{\mathrm{o}}_{I n}^{+} \\
\hat{\mathrm{o}}_{E l}+\hat{\mathrm{o}}_{E l}
\end{array}\right)_{G_{3}} \\
\left(\begin{array}{l}
\hat{\mathrm{o}}_{E l}^{+}+\underline{\mathrm{o}}_{E l}^{+} \\
\hat{\mathrm{o}}_{I n}+\hat{\mathrm{o}}_{I n}
\end{array}\right)_{\underline{G}_{3}}
\end{array}\right\}
$$

Note that the two possibilities $\left[\left(G_{3}\right)_{\mathrm{A}-\mathrm{rep}},\left(G_{3}\right)_{\mathrm{B}-\mathrm{rep}}\right]$ indicates always a different configuration by $\left(G_{1}, G_{2}\right)$ : therefore we can use indifferently both $\left(G_{3}\right)_{\mathrm{a}}$ and $\left(G_{3}\right)_{\mathrm{b}}$, 


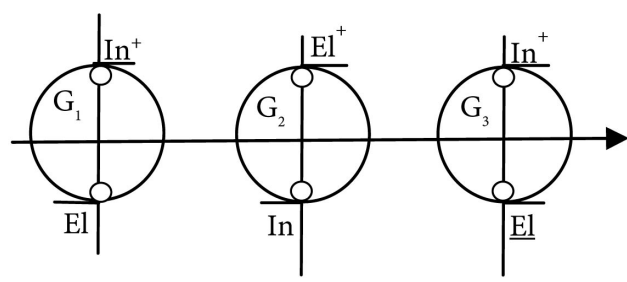

Figure 15. The unique possible combination for $\left(G_{1}, G_{2}, G_{3 A}\right)$.

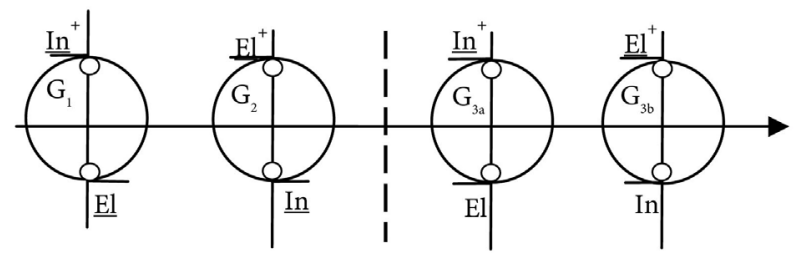

Figure 16. B-representation.

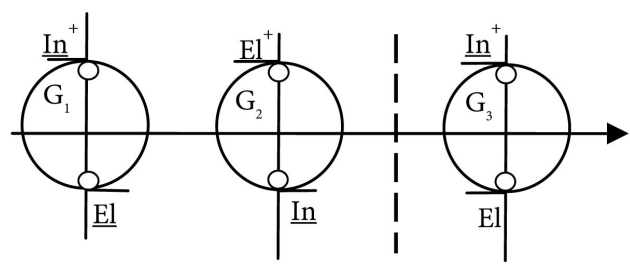

Figure 17. B-representation of base.

or a combination of them (see the 3.17a). We'll have the following representation (Figure 18 and Figure 19):

We call the $\mathrm{IQuO}_{(n=0)}$ as IQuO-0.

If we call the three internal degrees of freedom $\left(G_{i}\right)$ as "color charge" then we will define three colors [ $\left(G_{1} \equiv G_{\text {Red }}\right),\left(G_{2} \equiv G_{\text {Blu }}\right),\left(G_{3} \equiv G_{\text {Yellow }}\right]$ with representations (Figure 20):

And matrices

$$
\begin{gathered}
G_{R}=\left(\begin{array}{c}
\hat{\mathrm{o}}_{I n}^{+} \\
\hat{\mathrm{o}}_{E l}
\end{array}\right)_{R} \equiv\left(\begin{array}{cc}
0 & \hat{\mathrm{o}}_{I n}^{+} \\
\hat{\mathrm{o}}_{E l} & 0
\end{array}\right)_{R} \\
G_{B}=\left(\begin{array}{c}
\hat{\mathrm{o}}_{E l}^{+} \\
\hat{\mathrm{o}}_{I n}
\end{array}\right)_{B} \equiv\left(\begin{array}{cc}
\hat{\mathrm{o}}_{E l}^{+} & 0 \\
0 & \hat{\mathrm{o}}_{I n}
\end{array}\right)_{B} \\
G_{Y}=\left(\begin{array}{l}
\hat{\mathrm{o}}_{I n}^{+} \\
\hat{\mathrm{o}}_{E l}^{+}
\end{array}\right)_{Y} \equiv\left(\begin{array}{cc}
0 & \hat{\mathrm{o}}_{I n}^{+} \\
\underline{\hat{\mathrm{o}}}_{E l} & 0
\end{array}\right)_{Y}
\end{gathered}
$$

\subsection{The Electric Charge of Quarks}

As we know the abstract space to describe the hadrons, requires the transformation group SU(3). The generators of the group are the Gell-Mann matrices associated with three variables $\left(Y, T_{3}, Q\right)$. Remember that the multiplets existing in the hadrons are described by three physical quantities: hypercharge $(Y)$, the isospin $\left(T_{3}\right)$ and the electrical charge $Q$. We focus on the matrices $\lambda_{3}$ and $\lambda_{8}$, two generators of the group [2]: 


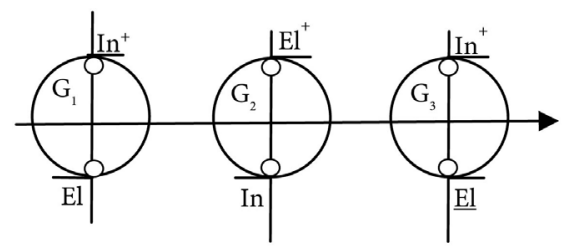

Figure 18. The unique possible combination.

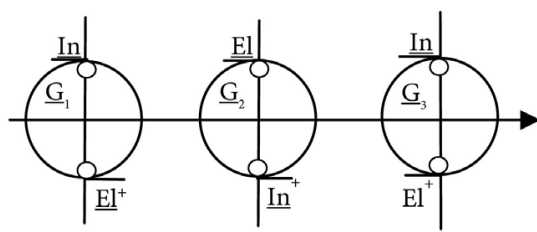

Figure 19. Conjugate representation of sub-oscillator.
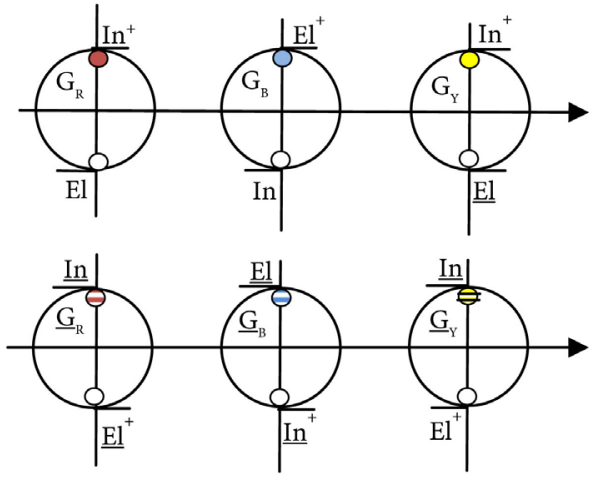

Figure 20. Sub-oscillators with color.

$$
\left\{\hat{\lambda}_{3}=\left(\begin{array}{ccc}
1 & 0 & 0 \\
0 & -1 & 0 \\
0 & 0 & 0
\end{array}\right) ; \quad \hat{\lambda}_{8}=\frac{1}{\sqrt{3}}\left(\begin{array}{ccc}
1 & 0 & 0 \\
0 & 1 & 0 \\
0 & 0 & -2
\end{array}\right)\right\}
$$

The $\lambda_{3}$ matrix is the projection in SU(3) of Pauli's matrix $\sigma_{3}$, giving [2] the values of $Q$ in the representation of the eigenvectors basic $\left(\Phi_{+}, \Phi_{-}\right)$. We recall (in Gell-Mann theory) the correlation between $Y$ and the matrix $\lambda_{8}$ :

$$
\hat{Y}=\left(\frac{1}{\sqrt{3}}\right) \hat{\lambda}_{8} \Rightarrow \hat{Y}=\left(\frac{1}{3}\right)\left(\begin{array}{ccc}
1 & 0 & 0 \\
0 & 1 & 0 \\
0 & 0 & -2
\end{array}\right)
$$

Giving the correlation between the charge $Q$ and the isospin $T_{3}$ and $Y$, we can consider the matrix product $\left[\lambda_{3}=\left(\lambda_{3}\right)(Y)\right]$

$$
\begin{aligned}
\hat{\lambda}_{3} & =\hat{\lambda}_{3} \hat{Y}=\left(\frac{1}{3}\right)\left(\begin{array}{ccc}
1 & 0 & 0 \\
0 & -1 & 0 \\
0 & 0 & 0
\end{array}\right)\left(\begin{array}{ccc}
1 & 0 & 0 \\
0 & 1 & 0 \\
0 & 0 & -2
\end{array}\right) \\
& =\left(\frac{1}{3}\right)\left(\begin{array}{ccc}
1 & 0 & 0 \\
0 & -1 & 0 \\
0 & 0 & 0
\end{array}\right)=\left(\begin{array}{ccc}
(1 / 3) & 0 & 0 \\
0 & -(1 / 3) & 0 \\
0 & 0 & 0
\end{array}\right)
\end{aligned}
$$


In conclusion, the matrix $\left(\lambda_{3}\right)$ can tell what values are allowed to charge $Q$ of a quark.

Then we think the IQuO of quark is expressed by matrix $(1 \times 3)$. In fact, if we recall the form of the wave function (see Figure 5) of the quantum oscillator with $(n=2)$ where there are three probability peak, then we can think that a $\mathrm{IQuOI}_{(n=2)}$ is made with three sub-oscillators. The superposition of two IQuO $\mathrm{I}_{(n=1)}$ or $\left(\mathbf{I}_{1(n=1)} \oplus \mathbf{I}_{2(n=1)}\right)$ builds an $\mathrm{IQuO}_{(n=2)}$ with three sub-oscillator but with the pairs $\left[(\bullet, \mathbf{o})_{1} ;\left((\bullet, \mathbf{o})_{2},(\bullet, \mathbf{o})\right)_{2} ;(\bullet, \mathbf{o})_{3}\right]$ where the index numbers indicate the sub-oscillators. This coupling can produce:

1) Two different $\mathrm{IQuO}$ (see the Equation (3.11))

2) An excited and unstable $\mathrm{IQuO} \mathrm{I}_{(n=2)}$

The b-possibility could be unstable because the central sub-oscillator is overpopulated in full semi-quanta $(\bullet)$. For have a stable IQuO in eigenstate $\mathrm{I}_{(n=2)}$ we need of overlapping an $\mathrm{IQuOI}_{(n=1)}$ with $\mathrm{I}_{(n=0)}$ : but this is possible only if we use quantum oscillators at semi-quanta and if the $\mathrm{I}_{(n=0)}$ is excited $\left(\mathrm{I}_{\left(n=0^{*}\right)}\right)$, that is we have an $\mathrm{IQuOI}_{(n=0)}$ composed by the pair $[(\bullet, \mathbf{o})]$. Note that to make couplings between sub-oscillators of two IQuO different it needs the exchange a full semi-quantum between the two respective component oscillators: this is allowed by a reciprocal intersection between the two IQuO (see Figure 21):

So, we need to define an operation $(\otimes)$ stating an appropriate combination of two matrices $[(2 \times 2)$ and $(2 \times 1)]$ generating one matrix $(2 \times 3)$. Using the matrix representation:

$$
\begin{aligned}
& {\left[\left(\begin{array}{cc}
{ }_{1}\left(\bullet_{e l}^{+}\right) & { }_{2}\left(\mathrm{o}_{i n}^{+}\right) \\
{ }_{1}\left(\mathrm{o}_{e l}\right) & { }_{2}\left(\bullet_{i n}\right)
\end{array}\right)_{(n=1)} \otimes\left(\begin{array}{l}
\left(\mathrm{o}_{e l}^{+}\right) \\
\left(\bullet_{i n}\right)
\end{array}\right)_{\left(n=0^{*}\right)}\right]=\left(\begin{array}{ccc}
{ }_{1}\left(\mathrm{o}_{e l}^{+}\right) & { }_{2}\left(\bullet_{e l}^{+}\right) & { }_{3}\left(\mathrm{o}_{i n}^{+}\right) \\
{ }_{1}\left(\mathrm{o}_{e l}\right) & { }_{2}\left(\bullet_{i n}\right) & { }_{3}\left(\bullet_{i n}\right)
\end{array}\right)_{\left(n=2^{*}\right)}} \\
& \text { or } \\
& \left(\begin{array}{ll}
{ }_{1}\left(\mathrm{o}_{e l}^{+}\right) & { }_{3}\left(\mathrm{o}_{i n}^{+}\right) \\
{ }_{2}\left(\bullet_{e l}^{+}\right) & { }_{2}\left(\bullet \bullet_{i n}\right) \\
{ }_{1}\left(\mathrm{o}_{e l}\right) & { }_{3}\left(\bullet_{i n}\right)
\end{array}\right)_{\left(n=2^{*}\right)}=\left(\begin{array}{l}
{ }_{1}\left(\mathrm{o}_{e l}^{+}\right)+{ }_{3}\left(\mathrm{o}_{i n}^{+}\right) \\
{ }_{2}\left(\bullet_{e l}^{+}\right)+{ }_{2}\left(\bullet_{i n}\right) \\
{ }_{1}\left(\mathrm{o}_{e l}\right)+{ }_{3}\left(\bullet_{i n}\right)
\end{array}\right)_{\left(n=2^{*}\right)} \equiv\left(\begin{array}{c}
{ }_{1}\left(\mathrm{o}_{e l}^{+}\right)+{ }_{3}\left(\mathrm{o}_{i n}^{+}\right) \\
{ }_{1}\left(\mathrm{o}_{e l}\right)+{ }_{3}\left(\bullet \bullet_{i n}\right) \\
{ }_{2}\left(\bullet_{e l}^{+}\right)+{ }_{2}\left(\bullet \bullet_{i n}\right)
\end{array}\right)_{\left(n=2^{*}\right)}
\end{aligned}
$$

where the index numbers indicate the sub-oscillators and where it is happened the change $(\bullet \Leftrightarrow \mathbf{o})$ between different sub-oscillators. The $\otimes$-operation thus implies an "elastic" attachment of sub-oscillators: $\left[(2)_{\text {sub }}+1_{\text {sub }}=3_{\text {sub }}\right]$.

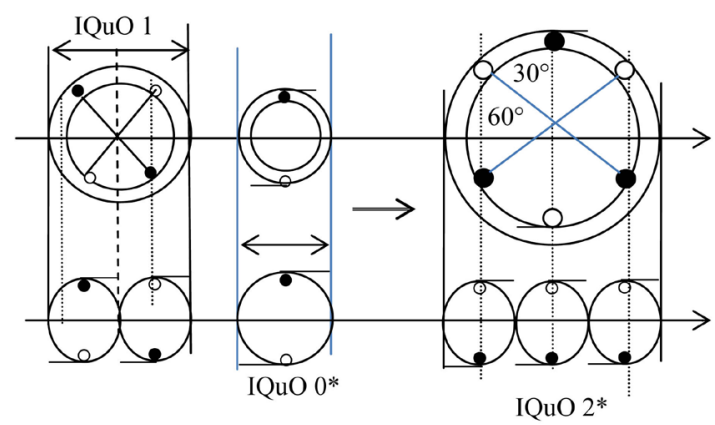

Figure 21. Superposition between two IQuO. 
Note that the result of the overlap between IQuO depends on the coupling of different modes.

We can reorganize the semi-quanta and elements of matrix obtaining the following $(3 \times 1)$ column matrix (whit the relative phases):

$$
((\Phi))_{c l}=\left(\begin{array}{c}
\left(\hat{o}_{e l} \mathrm{e}^{-i \rho}+\hat{\boldsymbol{\iota}}_{i n} \mathrm{e}^{-i \rho^{\circ}}\right) \\
\left(\hat{o}_{e l}^{+} \mathrm{e}^{-i \sigma}+\hat{\boldsymbol{\iota}}_{i n}^{+} \mathrm{e}^{-i \sigma^{\circ}}\right) \\
{ }_{c}\left(\hat{\boldsymbol{\iota}}_{e l}^{+} \mathrm{e}^{-i \alpha}+\hat{o}_{i n} \mathrm{e}^{-i \alpha^{\circ}}\right)
\end{array}\right)
$$

where index $\mathrm{c}$ indicates the couple of semi-quanta of the central sub-oscillator in Figure 21 and where [( $\left.\left.\alpha^{\circ}=\alpha+\pi / 2\right),\left(\rho^{\circ}=\rho-\pi / 2\right),\left(\sigma^{\circ}=\sigma \pm \pi / 2\right)\right]$.

It follows [2] the electric charge of $\mathrm{IQuO}_{\left(n=2^{*}\right)}$ :

$$
\begin{aligned}
Q_{c l} & =\left(\left(\Phi^{+}\right)\right)_{c l} \lambda_{3}((\Phi))_{c l} \\
& =\left(\begin{array}{c}
\left(\hat{o}_{e l}^{+} \mathrm{e}^{-i \rho}+\hat{\boldsymbol{\iota}}_{i n}^{+} \mathrm{e}^{-i \rho^{\circ}}\right) \\
\left(\hat{o}_{e l} \mathrm{e}^{-i \sigma}+\hat{\boldsymbol{\iota}}_{i n} \mathrm{e}^{-i \sigma^{\circ}}\right) \\
{ }_{c}\left(\hat{\boldsymbol{\iota}}_{e l} \mathrm{e}^{-i \alpha}+\hat{o}_{i n}^{+} \mathrm{e}^{-i \alpha^{\circ}}\right)
\end{array}\right)\left(\begin{array}{ccc}
1 / 3 & 0 & 0 \\
0 & -1 / 3 & 0 \\
0 & 0 & 0
\end{array}\right)\left(\begin{array}{c}
\left(\hat{o}_{e l} \mathrm{e}^{-i \rho}+\hat{\boldsymbol{\iota}}_{i n} \mathrm{e}^{-i \rho^{\circ}}\right) \\
\left(\hat{o}_{e l}^{+} \mathrm{e}^{-i \sigma}+\hat{\boldsymbol{\iota}}_{i n}^{+} \mathrm{e}^{-i \sigma^{\circ}}\right) \\
\left(\hat{\boldsymbol{\iota}}_{e l}^{+} \mathrm{e}^{-i \alpha}+\hat{o}_{i n} \mathrm{e}^{-i \alpha^{\circ}}\right)
\end{array}\right)=-\left(\frac{1}{3}\right)
\end{aligned}
$$

Considering the $\left(\left(\Delta \lambda_{3}\right)\right)$ as difference matrix of the matrices $\left[\left(\left(\lambda_{3}\right)\right),\left((Q) \equiv \lambda_{3}\right)\right]$ we obtain the other value $(Q=-2 / 3)$.

Note that the last pair of operators $\left(\mathrm{o}_{e l}^{+}, \bullet_{\text {in }}\right)_{c}$ is not involved in the calculus of the electric charge; this meaning that there is inside the quark another freedom degree.

As mentioned this couple is representing the color charge.

\subsection{The Isospin}

Taking up the idea of "internal" rotation of the phase [2] into IQuO, further "internal" rotations into a "composed" particle (as is a hadron) could be identified [1].

Remembering the electric charge connected to rotation of the phase of an IQuO (as "internal" rotation), we could connect the isospin (since it has similar structure to that of the "spin") to an "internal rotation" of "something" existing "inside" the hadrons.

As you well know, the transformations between hadrons show the existence of multiplets. Besides the "organization" of hadron in degenerate multiplets in mass and they internal transformations between hadrons prove that the hadrons are composed by "more elementary" particles called quarks. Thus the existing "rotation" inside a hadron could relate well to quarks; if the spin of a quark regards its spatial orientation, the isospin cannot regard to ulterior additional spatial orientations. Then we could assume that the "isospin rotation" could be correlated to the "rotation" of the internal current of "semi-quanta" that interconnects the quarks constituting a hadron. Remember the correlation between representative matrices of spin $(\sigma)$ and isospin (T). Besides, it can note that in Hadrons exists a correlation between the charge $Q$ and the isospin $T_{3}$. 
Remember that matrix $\sigma_{3}$ is also used to represent the projection $\left(T_{3}\right)$ of isospin $\mathrm{T}$ in the complex space $Q(2)$. In fact, it's:

$$
\hat{T}_{3}=\left(\frac{1}{2}\right) \hat{\lambda}_{3}=\left(\frac{1}{2}\right)\left(\begin{array}{ccc}
1 & 0 & 0 \\
0 & -1 & 0 \\
0 & 0 & 0
\end{array}\right)
$$

This formal analogy between $\sigma_{i} \mathrm{e}\left(T_{i}\right)$, as between $\sigma_{3}$ and $\left(T_{3}\right)$, cannot be random.

Also, note that the formal analogy between $\left(T_{3}\right)$ and the spin of a composite particle induces assign a value of $\left(T_{3}\right)$ also to the quarks.

It is recalled in fact the summative character of the isospin, then it follows that the isospin of a hadron is the sum of the values of isospin assigned to each individual quark.

Furthermore, the correlation between $T_{3}$ and $Q$, leads us to admit the existence of a correlation between the direction of rotation of the phase and the one of the isospin current.

This clearly happens in individual quarks, because there are the following associations:

1) Quark (u): $Q(>0) \leftrightarrow\left(T_{3}\right)^{\prime}=(+1 / 2)$

2) Quark (d): $Q(<0) \leftrightarrow\left(T_{3}\right)^{\prime}=(-1 / 2)$

\section{Note that $Q$ and $T 3$ have the same sign}

Thus, ultimately, we can assign three intrinsic "rotations" to IQuO-quark:

1) Internal phase rotation ( $Q$ sign)

2) Internal Isospin rotation $\left(T_{3}\right.$ sign $)$

3) External Spatial rotation of spin ( $\sigma$ sign)

This may seem at odds with the "spatially local" aspect assigned to the elementary particle (like quarks and leptons), in perfect agreement with the relativistic quantum theory. However, considering what already talked about the rotations inside to hadron and taking into account the phenomenology of the interactions between hadron, it can become acceptable assume the following hypothesis of Structure: even if an elementary particle proves to be a "local unit" in space for an external observer, it may instead show the existence of an "internal" space because described by variations of physical quantities that can be identified as "internal" rotations showing an "internal structure" of couplings of IQuO.

If the isospin $\left(T_{h}\right)$ assigned to a hadron identifies an internal current (semi-quanta current or "gluonic" current) then the isospin $\left(T_{q}\right)$ assigned to a quark identically detects a semi-quanta current that identify a "path" between elementary "internal points of oscillation", though always considering a quark as an "oscillating" unique physical system.

A quark, though maintaining the external aspect of an energy quantum in a space point, could instead be constituted by an internal structure of "virtual" "IQuO called" Sub-IQuO". This "virtual" internal structure of a quark has a descriptive index that we individualize in the Hypercharge $Y$. The latter, as well as 
being introduced to differentiate the hadron multiplets, is assigned to an individual quark, reinforcing the idea of its sub-structure related to different configurations of coupling of sub-IQuO that make it.

\subsection{The Hypercharge $Y$}

Another support for this hypothesis of Structure is identifiable in the correlations between the "internal" rotations (those of phase and isospin) during the hadron interactions (you see Clebsch-Gordon coefficients).

It was noted that $Q$ and $T_{3}$ have the same algebraic sign; this implies that the isospin current between "Sub-IQuO" constituents of a quark follows the same "direction" of rotation of the phase of each Sub-IQuO. It's evident that the structure of sub-IQuO (you see $Y$ hypercharge) has to be compatible with the correlation $\left(Q \leftrightarrow \rightarrow T_{3}\right)$. It follows that has to exist a connection $\left(Q \leftrightarrow \rightarrow T_{3} \leftrightarrow\right.$ Y).

The relation of Gell-Mann expresses this connection: [ $\left.Y=2\left(Q-T_{3}\right)\right]$

The correlation ( $Q \leftrightarrow \rightarrow T_{3} \leftrightarrow \rightarrow Y$ ) made manifest both in the correlation between the matrices $\lambda_{3}$ e $\lambda_{8}$, as we shall see later, both in the combination of the respective generators during interactions.

Underlining the correlation of the sign of $Q$ (direction of phase rotation) with the sign of $\mathrm{T}$ (direction of current rotation) and with a value of $Y$ (structure by couplings), one derives that the electric charge values are connected with the structure of quarks. It's evident that this connection determines in hadron interactions the various probabilities of the combination of isospin couplings as the probabilities of the electric charge values of the quarks. This also suggests that the non-integer value of the charge of a quark is an expression of probabilistic aspects associated with the structure of couplings of the sub-IQuO which compose the quark. If the direction of phase rotation of a particle-IQuO gives to us the sign of the electric charge, its value can instead be placed in relation with the probability that a photon detects the quanta of the charged particle which has a particular structure of sub-IQuO. If into electron this probability has a value equal to one, the probability in quarks has not an integer value because the triangular structure of a quark has a number of quanta minor that the number of sides. If inside a quark there are more sub-IQuO, the photon in coupling with one of sub-IQuO components cannot find the representative quantum of the quark. For the definition of statistical probability, if a quark may be no seen by a single photon, then it will be not seen by an infinite number of photons. How to say that a quark would be invisible to single photon.

However, if the single quark cannot be seen, a hadron composed by more quarks can be detected only if the sum of the probability of finding the quantum has a value of a unit or the sum of the electric charges of the quarks of a hadron reaches the value of a unit. This is because two or three quarks bound to become a single unit (or a single center diffuser) for couplings with photons.

The information of the non-integer value of the electric charge of a quark can 
also be found in the Gell-Mann matrix $\lambda_{8}$, which is an expression of the connection $(Q \leftrightarrow \rightarrow Y)$. In fact, (see the equation 3.19) it is no random coincidence that the matrix $\lambda_{8}$ precisely commutes with the $\lambda_{3}$ (you see $\sigma_{3}$ ). In [1] so note that the eigenvalues of $Y$ are related to the values of electric charge $Q$. Obviously, everything is perfectly in accord with the relation: $\left[Y=2\left(Q-T_{3}\right)\right]$ ).

Therefore, we think that the quark is given by one "triangular" structure of three "sub-IQuO" (see IQuO-vertices) coupled to each other through IQuO chains (sides) in which flows a current of semi-quanta. The variable descriptive of the semi-quanta current is the Isospin $(T)$ whose projection $\left(T_{3}\right)$ is connected to the rotation direction of the current, we have the following correspondence:

- Clockwise $\Leftrightarrow T_{3}=-1 / 2$

- Anticlockwise $\Leftrightarrow T_{3}=+1 / 2$

The variable descriptive of the phase rotation in IQuO-vertices is electric charge $Q$ :

- Clockwise $\Leftrightarrow(-Q)$

- Anticlockwise $\Leftrightarrow(+Q)$

By triangular structure of the couplings, it follows that the direction of rotation of the semi-quanta current along the sides shall be concordant with the direction of rotation of the phase of IQuO-vertices. This expresses the correlation ( $Q \Leftrightarrow T_{3}$ ). Also to its structure, it shall be associated with descriptive variable that we believe it is the $Y$ (hypercharge). It is evident that the probability of finding the quantum of a particle inside of a structure depends on this latter. As the value of the electric charge $Q$ has been associated with the probability of detecting the representative quantum of the quark, it follows the ( $Q \Leftrightarrow Y$ ) correlation. The correlation $\left(Q \Leftrightarrow Y \Leftrightarrow T_{3}\right)$ is now:

- Clockwise $\Leftrightarrow Q=[(-1 / 3,-2 / 3)]$

- Anticlockwise $\Leftrightarrow Q[(+1 / 3,+2 / 3)]$

We ask in what way the $Y$ is related to the structure. A triangular structure of IQuO-V coupled suggests that get involved more chains of IQuO and therefore it should exist the possibility to realize a coupling "transverse" between separate chains. Chains involved in the triangular closure defining a quark can be in a number of two or three at the most (you see the Figure 7 and Figure 8).

For everything that we affirmed up to now, we can say that the hypercharge $Y$ is the indicating physical variable the triangular structure of coupling of Sub-IQuO vertices to which we associate the value $1 / 3$ as to say that there is one particle-quantum on three vertices. Also, noting that a triangle in the mirror $(\mathrm{P})$ undergoes inversion right - left, we assign the value of the hypercharge $Y=-1 / 3$ to the antimatter. We note that the direction of rotation of any rotating object reflected in the mirror is reversed, thus both the isospin both the electrical charge changing the sign: $\left[\left( \pm T_{3} \rightarrow \mp T_{3}\right)\right]$. It obvious then that you must also have a reversal of the value of hypercharge $( \pm Y \rightarrow \mp Y)$. We have thus explained the physical meaning about what we describe with electric charge $Q$, with isospin $T$ and hypercharge $Y$. 


\section{The Structure of Coupled Oscillators}

\subsection{The Coupling of Chains}

If to an only oscillator (quantum and classic) we assign an elastic component (see $\boldsymbol{a}_{e l}$ in quantum oscillator) which represents the elastic tension $(k)$ acting on the "mass" $(M)$ of oscillator (see $\boldsymbol{a}_{i n}$ ), to the chain of coupled oscillators we assign [1] the same elastic tension $(k)$ between oscillators (see Figure 1). The same aspect is in the two sub-oscillators which component the IQuO. In summary: so as two sub-oscillators couple for making an only IQuO, then it is possible that more IQuO couple for making up a "chain" of coupled quantum oscillators. To chain (see Figure 1 ) is assigned an elastic tension $T:[k \Delta l]$. We think that can be possible the following representation of chain (Figure 22):

Note (see Figure 8 and Figure 9(a)) that the semi-quanta oscillate $(\bullet)$ inside each IQuO: the couple $((\bullet),(\bullet))$ would oscillate always only between the points $(\mathrm{A}, \mathrm{C})$ in IQuO 1. Nevertheless, it is necessary even that the couple $((\bullet),(\bullet))$ propagates along the $\mathrm{X}$-axis: the propagation from IQuO-1 to the next (IQuO-2) needs so to oscillate with variable frequency $(\omega)$. To have both propagation and oscillation of couple $((\bullet),(\bullet))$ it needs a superposition with another chain, but shifted (of $\pi / 2$ ): this could allow an oscillation of the full semi-quantum couple $((\bullet),(\bullet))$ inside a sub-oscillator of IQuO and after the propagation along axis X. In first we report the figure of two IQuO (you see Figure 23) in a particular overlap:

We note that two IQuO overlap and sharing the sub-oscillator central. Two IQuO can exchange energy only in the point $\mathrm{C}$ of the central sub-oscillator. The operation of superposition is indicated by $(\underline{\oplus)}$ : $(\underline{\oplus})$ is the overlap of two IQuO involving an only sub-oscillator of two IQuO (see Figure 23).

We note the exchange $\left[\left(a_{i n}\right)(\bullet) \rightarrow\left(A_{i n}\right)(\mathrm{o})\right]$ happens in point $\mathrm{C}$ of the configuration; these exchanges make oscillate the couple $((\bullet),(\bullet))$ inside central oscillator just once because after it happens the passing to first sub-oscillator of the 2-IQuO.

In this way, the quantum $((\bullet),(\bullet))$ can propagate with oscillations along the chain and so to constitute a "field chain". It follows any field can be represented by a set of "field chains". In the case of closed chains (see the sides of a triangular structure) then the superposition of two chains in each side is the fundamental condition for having the oscillating propagation of couple $((\bullet),(\bullet))$ along the sides. It is evident that this determines even the "isospincurrent" inside the triangular structure of quark.

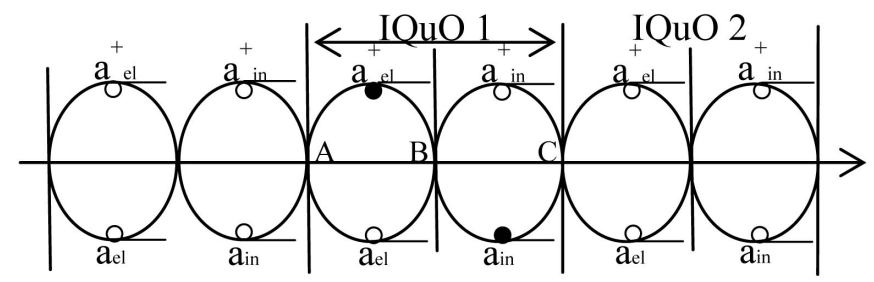

Figure 22. IQuO chain. 


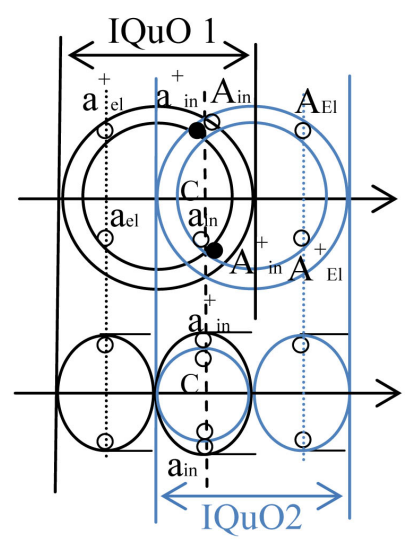

Figure 23. Two IQuO in overlap.

\subsection{The Quark Structure}

A structure of IQuO coupled oscillators can even involve at least two or most distinct chains of IQuO. It's evident that the coupling between distinct chains of IQuO can be built with the help of "transversal" chains (Figure 24) which "intersect" them and form so an additional coupling, you see the massive coupling (see par. 1). Graphically:

The transversal IQuO represent the "massive coupling". So the structure in Figure 24 will be represented (see Equation (3.10)) by matrix (see Figure 11):

$$
[\Psi]_{-} \equiv\left(\begin{array}{c}
{\left[\begin{array}{cc}
\left(\mathrm{o}_{e l}\right) & \left(\bullet_{i n}\right) \\
\left(\bullet_{e l}^{+}\right) & \left(\mathrm{o}_{i n}^{+}\right)
\end{array}\right]_{\text {mass }}} \\
\left(\begin{array}{ll}
\left(\mathrm{o}_{e l}\right) & \left(\mathrm{o}_{i n}\right) \\
\left(\mathbf{\bullet}_{e l}^{+}\right) & \left(\underline{\mathrm{o}}_{i n}^{+}\right)
\end{array}\right) \\
\left(\begin{array}{ll}
\left(\mathrm{o}_{e l}\right) & \left(\mathrm{o}_{i n}\right) \\
\left(\mathbf{\bullet}_{e l}^{+}\right) & \left(\underline{\mathrm{o}}_{i n}^{+}\right.
\end{array}\right)
\end{array}\right)=\left(\begin{array}{c}
{\left[\begin{array}{c}
\mathrm{o}_{e l}+\bullet_{i n} \\
\bullet_{e l}^{+}+\mathrm{o}_{i n}^{+}
\end{array}\right]} \\
\left(\begin{array}{c}
\mathrm{o}_{e l}+\mathrm{o}_{i n} \\
\mathbf{\bullet}_{e l}^{+}+\underline{\mathrm{o}}_{i n}^{+}
\end{array}\right) \\
\left(\begin{array}{c}
\mathrm{o}_{e l}+\mathrm{o}_{i n} \\
\mathbf{\bullet}_{e l}^{+}+\underline{\mathrm{o}}_{i n}^{+}
\end{array}\right)
\end{array}\right)
$$

This is a column matrix; the square brackets will indicate the IQuO of massive coupling. Note that inside in any chain with massive coupling there are IQuO of type "Boson" (see the Figure 9).

An elementary "geometric structure" involving "three" chains of IQuO can be given by "isosceles triangles" (you see Figure 25):

The elementary coupling between the distinct IQuO chains [(1), (2), (3)] non-overlapping can be realized by means of transverse quantum oscillators $\left[\left(I_{A B}\right),\left(I_{A C}\right),\left(I_{B C}\right)\right]$, which joining three IQuO-Vertices (IQuO-V) $\left[\left(I_{A}\right),\left(I_{B}\right),\left(I_{C}\right)\right]$ placed each in one of three chains involved.

An IQuO-V in order to belong to a chain and to the triangular structure of the couplings has to be an $\mathrm{IQuO}_{\left(n=2^{*}\right)}$, with three sub-oscillators but with only three full semi-quantum $(\bullet))$. This is because its sub-oscillators can so overlap to the sub-oscillators of the junction IQuO or IQuO-J (the three IQuO: $\mathrm{I}_{\mathrm{AC}}, \mathrm{I}_{\mathrm{BC}}, \mathrm{I}_{\mathrm{AB}}$ ) connecting the three vertices. To achieve this, in analogy with the physical system "springs-spheres", it is necessary to have (Figure 26): 


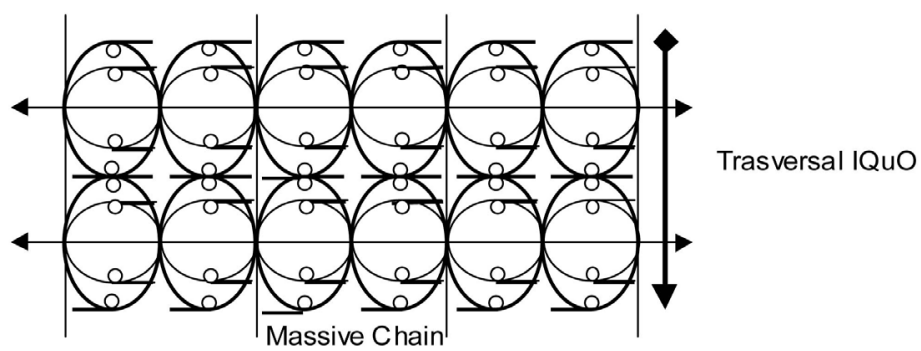

Figure 24. The "massive coupling".

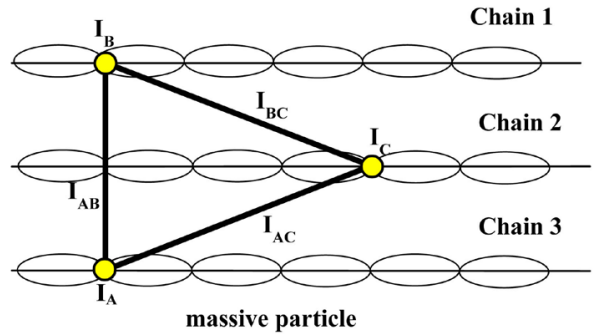

Figure 25. Three coupled chains.

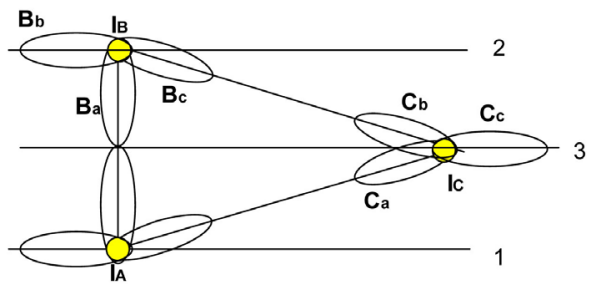

Figure 26. The vertex-IQuO in a triangular structure.

Where $\left[\mathrm{A}_{\mathrm{i}}, \mathrm{B}_{\mathrm{i}}, \mathrm{C}_{\mathrm{i}}\right]$ is the component sub-oscillators of an IQuO-V.

Note well that in this figure for clarity are not shown the connecting IQuO-J.

As we have already said, the geometric coupling structure can express spatial "orientation" or "spin" and, the same time, to propagate in space. There are more descriptive possibilities:

1) The vertices $\left(I_{B}\right)$ and $\left(I_{A}\right)$, you see the Figure 26 , are detached from the respective chains ( 1 and 2 ) while the vertex $I_{C}$ is connected to the chain 3 . In this case the structure can rotate around the direction of the chain 3 , where it is in moving.

2) Each vertex moves along its own chain and the spin will be given by its particular IQuO vertices of the structure.

Nevertheless, we could consider these two representations as equivalents.

The representative matrix of IQO-V (with $n=2^{\star}$ because it is composed by 3 sub-oscillators with 3 full semi-quanta $(\bullet))$ will be (see (3.23)):

$$
\left\{\Phi_{c l} \equiv\left(\begin{array}{cc}
\left(\mathrm{o}_{e l}\right) & \left(\bullet_{i n}\right) \\
\left(\bullet_{e l}^{+}\right)_{c} & \left(\mathrm{o}_{i n}\right)_{c} \\
\left(\mathrm{o}_{e l}^{+}\right) & \left(\bullet_{i n}^{+}\right)
\end{array}\right)_{V} ; \quad \Phi_{c l} \equiv\left(\begin{array}{cc}
\left(\bullet_{e l}^{+}\right) & \left(\underline{\mathrm{o}}_{i n}^{+}\right) \\
\left(\underline{\mathrm{o}}_{e l}\right)_{c} & \left(\underline{\bullet}_{i n}^{+}\right)_{c} \\
\left(\bullet_{e l}\right) & \left(\underline{\mathrm{o}}_{i n}\right)
\end{array}\right)_{V}\right\}
$$


where the second row describes the central sub-oscillator (see "c" index) of a quantum oscillator in one of several possible configurations.

So, an any $\mathrm{IQuO}_{\left(n=2^{*}\right)}$ is represented by a column matrix with three elements.

In matrix form [2] and renewing the phase, we conjecture (see Equation (3.23)) that:

$$
((\Phi))_{c l}=\left(\begin{array}{c}
\left(\hat{o}_{e l} \mathrm{e}^{-i \rho}+\hat{\boldsymbol{\bullet}}_{i n} \mathrm{e}^{-i \rho^{\circ}}\right) \\
\left(\hat{\boldsymbol{\bullet}}_{e l}^{+} \mathrm{e}^{-i \alpha}+\hat{o}_{i n} \mathrm{e}^{-i \alpha^{\circ}}\right)_{c} \\
\left(\hat{o}_{e l}^{+} \mathrm{e}^{-i \sigma}+\hat{\boldsymbol{\bullet}}_{i n}^{+} \mathrm{e}^{-i \sigma^{\circ}}\right)
\end{array}\right) \equiv{ }_{3}\left(\begin{array}{c}
\left(\hat{o}_{e l} \mathrm{e}^{-i \rho}+\hat{\boldsymbol{\iota}}_{i n} \mathrm{e}^{-i \rho^{\circ}}\right) \\
\left(\hat{o}_{e l}^{+} \mathrm{e}^{-i \sigma}+\hat{\boldsymbol{\iota}}_{i n}^{+} \mathrm{e}^{-i \sigma^{\circ}}\right) \\
\left(\hat{\boldsymbol{o}}_{e l}^{+} \mathrm{e}^{-i \alpha}+\hat{o}_{i n} \mathrm{e}^{-i \alpha^{\circ}}\right)
\end{array}\right)
$$

where $\left[\left(\alpha^{\circ}=\alpha+\pi / 2\right),\left(\rho^{\circ}=\rho-\pi / 2\right),\left(\sigma^{\circ}=\sigma \pm \pi / 2\right)\right]$.

If three transversal chains crossing reciprocally it is possible to form three IQuO-V. Having the oblique chains it is possible that there are some shifts in respective IQuO-V components: there is the possibility that an $\mathrm{IQuO}-\mathrm{V}_{(n=2)}$ is formed but with three sub-oscillators not aligned. This can generate component sub-oscillators in vacuum state $(n=0)$ :

$$
\left[\mathrm{IQuO}_{\left(n=2^{*}\right)} \Rightarrow 3 \mathrm{IQuO}_{\left(n=0^{*}\right)}\right]
$$

Nevertheless, to keep the three chain connected then it is needed that in the longer sides-chains there are $\mathrm{IQuO}_{\left(n=2^{*}\right)}$. So note that the two oblique IQuO-J should be longer than that of the base: if IQuO-side ${ }_{\left(n=2^{*}\right)}$ then IQuO (base) $)_{\left(n=1^{\star}\right)}$.

The IQuO of connecting (IQuO-J) should be so constituted by a central sub-osc. and two lateral sub-oscillators: these last are overlapping of the lateral sub-oscillators of the IQuO-V. This could cause a discontinuity in the flow of the semi-quanta within IQuO belonging to quark. Instead, it is needs to have a continuous flow of semi-quanta long the sub-oscillators (you see the isospin), namely all sub-oscillators must be of the same type and to have the same number of semi-quanta. So, to achieve a continuum in the flow of semi-quanta it is necessary to have a side with double chain or, in the same way, it is necessary to double all the sub-oscillators which compose the connecting lines of vertices (see Figure 27):

For any side there will be a superposition of two IQuO:

Side AB: $\left[\left(\mathrm{I}_{\mathrm{A}}\right)_{(n=2)} \cap\left(\mathrm{I}_{\mathrm{AB}}\right)_{(n=1)} \cap\left(\mathrm{I}_{\mathrm{B}}\right)_{(n=2)}\right]$

Side BC: $\left[\left(\mathrm{I}_{\mathrm{B}}\right)_{(n=2)} \cap\left(\mathrm{I}_{\mathrm{BE}}\right)_{(n=2)} \cap\left(\mathrm{I}_{\mathrm{CH}}\right)_{(n=2)} \cap\left(\mathrm{I}_{\mathrm{C}}\right)_{(n=2)}\right]$

Side CA: $\left[\left(\mathrm{I}_{\mathrm{C}}\right)_{(n=2)} \cap\left(\mathrm{I}_{\mathrm{IL}}\right)_{(n=2)} \cap\left(\mathrm{I}_{\mathrm{LA}}\right)_{(n=2)} \cap\left(\mathrm{I}_{\mathrm{C}}\right)_{(n=2)}\right]$

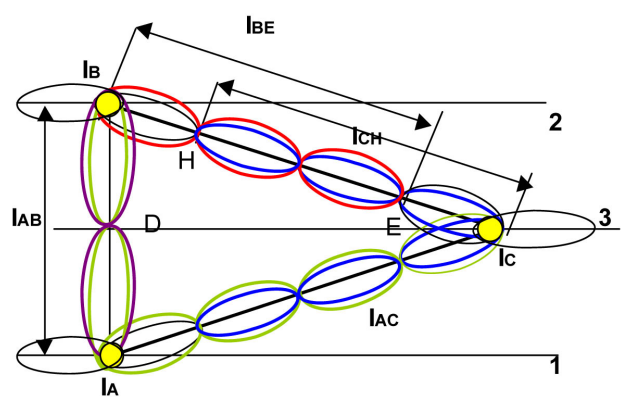

Figure 27. Any side is an oblique "double chain". 
Important: note that in this way a quark is like composed by attached individual sub-oscillators (see Figure 27). Recall: $\left[\mathrm{I}_{(n=0)} \Leftrightarrow 1\right.$ sub-osc.].

Thus we'll say that a quark is a structure of sub-oscillators.

Besides, during the quark formation, the coupling between two vertices $(A, B)$ and the chains $(1,2)$ could break: it could happen that the two chain (1 and 2) can break away from the IQuO-V $\left(\mathrm{I}_{\mathrm{A}}, \mathrm{I}_{\mathrm{B}}\right)$. The quark structure could remain instead intact with the possibility of propagation along axis $\mathrm{X}$ (3-chain). As we said, this possibility allows, for symmetry, a "spin" of the quark around axis $\mathrm{X}$ (3-axis). The quark can tie with other quark along a side [4] as the BC, forming a hadron; in this case, the $\mathrm{IQuO}_{\mathrm{A}}$ remains free and, for rotational symmetry, one can associate a rotation (spin) of the triangular structure of quark around to (BC)-axis, which becomes the chain of propagation of new hadron.

Note that in forming a quark the elastic tension along sides can be different in the some points, see the Figure 28:

The $k$-elastic tension of sub-oscillator of the $\mathrm{IQuO}\left[\left(\mathrm{I}_{\mathrm{CH}}\right),\left(\mathrm{I}_{\mathrm{C}}\right),\left(\mathrm{I}_{\mathrm{FC}}\right)\right]$ is more rigid $\left(k_{\mathrm{C}}>k_{\mathrm{B}}\right)$ than in $\mathrm{I}_{\mathrm{B}}$ and $\mathrm{I}_{\mathrm{A}}$, because the $\mathrm{C}$-vertex is attacked to the chain while the vertexes $A$ and $B$ are now free. Note that when we apply the same force to the ends of two attached springs, but different in elastic tension, then we will see that the spring with the smaller elastic tension is longer than the spring with the greater elastic tension. In this way the sub-oscillators [(BH), (B), (BD), (AD), $(\mathrm{A}),(\mathrm{AF})]$ are stretched. If $(\mathrm{BH}=1(1 / 2))$, then we could admit:

$$
\begin{aligned}
B C & \equiv\left\{\left(I_{B E}\right) \underline{\oplus}\left(I_{C H}\right)\right\} \equiv\left[B H\left(I_{B}\right)+C H\left(I_{C H}\right)\right] \\
& =\left(\frac{l}{2}\right)+\left(\frac{l \sqrt{5}}{2}\right)=l\left(\frac{1+\sqrt{5}}{2}\right)=l \phi
\end{aligned}
$$

where $(\phi)$ is the "aureus" number. Besides

$$
\left\{\begin{array}{c}
A B \equiv\left\{\left(I_{A B}\right) \oplus\left(I_{A}\right)\right\} \equiv\left[A D\left(I_{A}\right)+D B\left(I_{B}\right)\right]=l\left(\frac{1}{2}\right)+l\left(\frac{1}{2}\right)=l \\
\left(I_{A B}\right)_{(n=1)}=\left(\frac{l^{\prime} \sqrt{3}}{2}\right)
\end{array}\right\}
$$

For compatibility has to be the case that

$$
l^{\prime}=\left(\frac{2}{\sqrt{3}}\right) l
$$

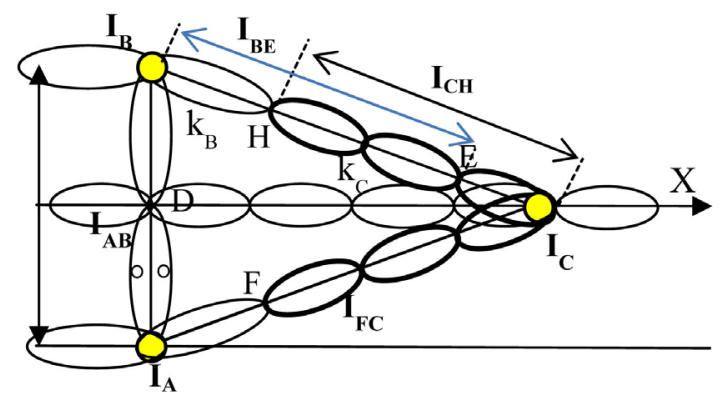

Figure 28. Elastic tensions $(k)$ in quark. 
it follows that $\left[\mathrm{I}_{\mathrm{AB}}=1\right]$ and then we will have that $\left[\left(\mathrm{I}_{\mathrm{BC}}\right) /\left(\mathrm{I}_{\mathrm{AB}}\right)=\varphi\right]$.

This aureus ratio defines an "aureus triangle" with vertex angle of $\left(72^{\circ}\right)$ and these structures of coupled IQuO will define the "aureus structures" of the quarks

\subsection{The Quark Structure $(u, d)$}

Now we formulate the following hypothesis (see also [3] [4]): an "aurea" triangular structure of coupled quantum oscillators $(\mathrm{IQuO})$ with vertex angle of $\left(72^{\circ}\right)$ corresponds to the quark (d). From the (Figure 27) and (Figure 2) we derive, shortly and schematically, that (see Figure 29):

Where $\left(\lambda_{d}\right)$ is the wavelength Compton of d-quark. Recalling the pentagon (Figure 2), we assume that the "aureae" lateral triangles could represent the quark (u) (see [1] [4]). The graphics "relation" between the quark (d) and (u) will be (Figure 30):

Where $\left(\lambda_{u}\right)$ is the wavelength Compton of u-quark, with C-vertex angle of $\left(104^{\circ}\right)$.

We have already said that the quark is an overlap of IQuO-V and transversal-IQuO(see Figure 27): $\left[\left(\mathrm{I}_{\mathrm{A}}(\underline{\oplus}) \mathrm{I}_{\mathrm{AB}}(\underline{\oplus}) \mathrm{I}_{\mathrm{B}}(\underline{\oplus}) \mathrm{I}_{\mathrm{BC}}(\underline{\oplus}) \mathrm{I}_{\mathrm{C}}(\underline{\oplus}) \mathrm{I}_{\mathrm{CA}}\right)\right]$.

Note that the $\mathrm{IQuO}$-side $\left[\mathrm{I}_{\mathrm{AB}}, \mathrm{I}_{\mathrm{BC}}, \mathrm{I}_{\mathrm{CA}}\right]$ are composed (see Figure 27) by the intersection of two $\mathrm{IQuO}_{\left(n=2^{*}\right)}$.

This represent the overlapping state of IQuO of a quark-structure: we speak about a structure in "entanglement state" because any component IQuO is not separated by the others and any elastic perturbation in a single IQuO propagate

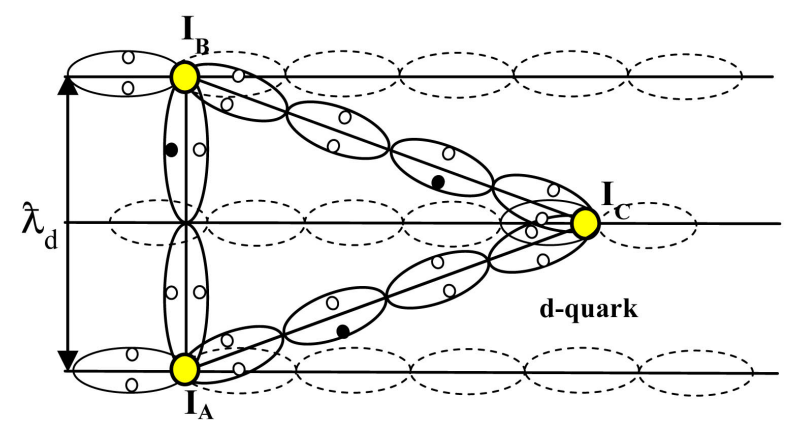

Figure 29. The d-quark form.

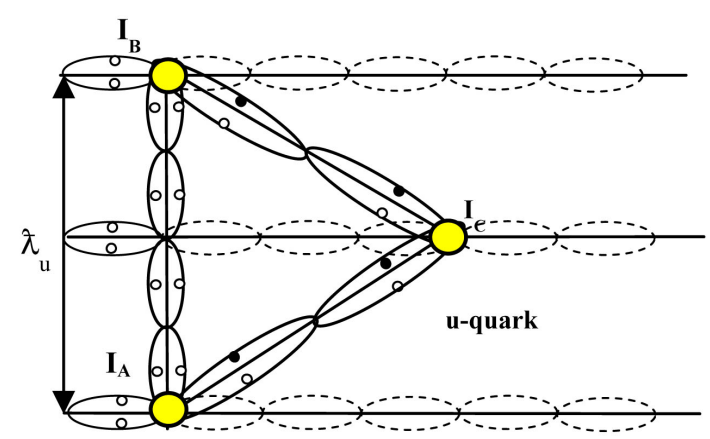

Figure 30. The u-quark. 
in all structure with phase velocity $\left(v_{\varphi} \geq c\right)$. Omit for the time being the junction-IQuO, then the representative state can be by overlap of three IQO-V $\left[\left(\mathrm{I}_{\mathrm{A}}\right.\right.$ $\left.\left.(\underline{\oplus}) \mathrm{I}_{\mathrm{B}}(\underline{\oplus}) \mathrm{I}_{\mathrm{C}}\right)\right]$ :

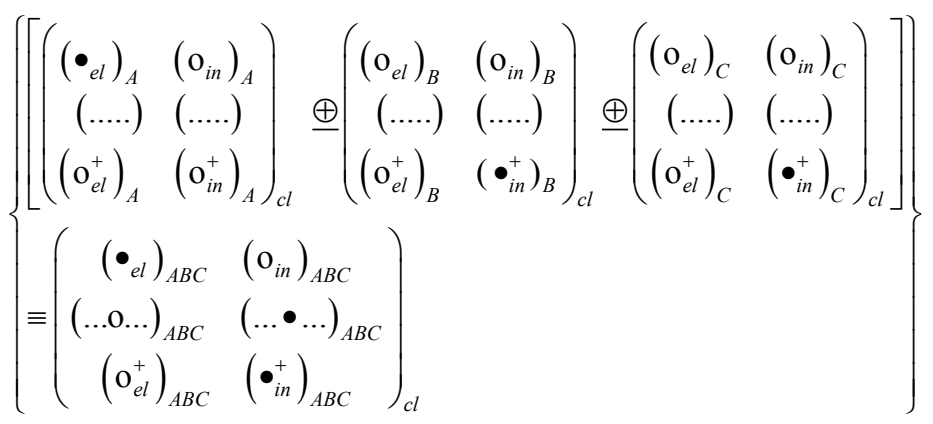

With representation (Figure 31).

\section{The Gluons}

\subsection{The Gluon}

We can think that there are different possibilities of combinations between IQuO-0 or sub-oscillator $G_{i}$. We can combine $\left[\left(\underline{G}_{1}, \underline{G}_{2}\right)\right]$ in overlap (see Figure $32)$.

Note that the two IQuO-0 does not combine for generate an any IQuO normal $\left(\mathrm{IQuO}_{(n=1)}\right)$; then we could believe to the possibility of having a particular superposition of two sub-oscillators which origin a particular form of IQuO, defined IQuO-G. That is an $\left(\mathrm{IQuO}_{(n=1)}\right)$ with only one sub-oscillator.

This new possibility of combination in a superposition of IQuO-0 will be indicated with $(\underline{\underline{\oplus}})$.

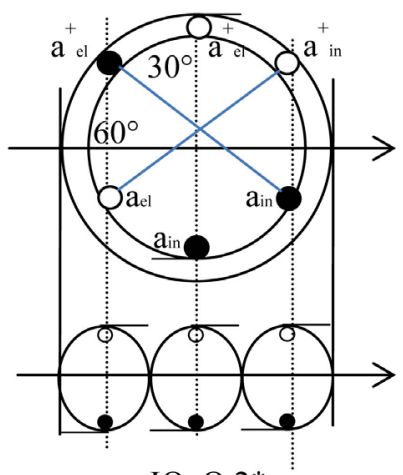

$\mathrm{IQuO} 2^{*}$

Figure 31. Configuration of internal "sub-IQuO" to quark.

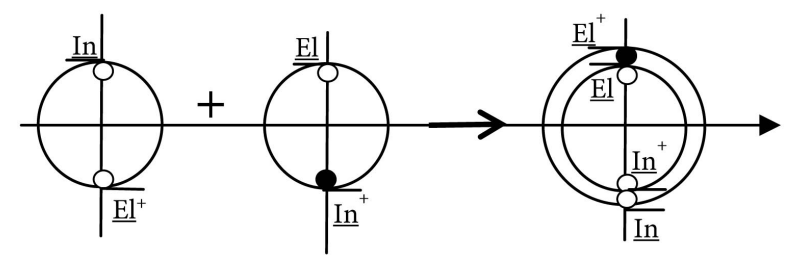

Figure 32. Formation of a gluon. 
If we combine $\left[G_{1} \stackrel{\oplus}{\underline{G}} \underline{G}_{1}\right]$ then we could admit the following representation (Figure 33):

In matrices:

$$
G_{R} \stackrel{\oplus}{\underline{G}} \underline{G}_{R}=\left(\begin{array}{c}
\hat{\mathrm{o}}_{I n}^{+} \\
\hat{\mathrm{o}}_{E l}
\end{array}\right)_{R} \stackrel{\oplus}{=}\left(\begin{array}{l}
\hat{\mathbf{o}}_{E l}^{+} \\
\underline{\hat{\mathrm{o}}}_{I n}^{+}
\end{array}\right)_{\underline{R}}={ }_{1}\left(\begin{array}{c}
\hat{\mathrm{o}}_{I n}^{+}+\hat{\mathbf{o}}_{E l}^{+} \\
\hat{\mathrm{o}}_{E l}+\underline{\hat{\mathrm{o}}}_{I n}^{+}
\end{array}\right)_{R \underline{R}}
$$

or

$$
G_{R} \stackrel{\oplus}{=} \underline{G}_{R} \equiv\left(\begin{array}{cc}
0 & \hat{\mathrm{o}}_{I n}^{+} \\
\hat{\mathrm{o}}_{E l} & 0
\end{array}\right)_{G_{R}} \stackrel{\oplus}{=}\left(\begin{array}{cc}
\hat{\boldsymbol{o}}_{E l}^{+} & 0 \\
0 & \underline{\hat{o}}_{I n}^{+}
\end{array}\right)_{\underline{G}_{R}}={ }_{1}=\left(\begin{array}{cc}
\hat{\boldsymbol{o}}_{E l}^{+} & \hat{\mathrm{o}}_{I n}^{+} \\
\hat{\mathrm{o}}_{E l} & \underline{\hat{o}}_{I n}^{+}
\end{array}\right)_{R \underline{R}}
$$

where the index 1-number indicates one state with only one sub-oscillator. The two matrices are equivalent. This configuration does not represents an IQuO but an overlap of two sub-oscillators in quantum entanglement (see the operation $\underline{\underline{\oplus}}$. We conjecture these possibilities as "Gluons". The possible combinations, with $(\underline{\underline{\oplus}})$, of matrices are:

$$
\left(\begin{array}{lll}
R \underline{R} & R \underline{B} & R \underline{Y} \\
B \underline{R} & B \underline{B} & B \underline{Y} \\
Y \underline{R} & Y \underline{B} & Y \underline{Y}
\end{array}\right)
$$

One some representations (Rㅡ) can be (Figure 34):

With representative matrix

$$
G_{R \underline{B}}=\left(\begin{array}{c}
\hat{\mathrm{o}}_{I n}^{+} \\
\hat{\mathrm{o}}_{E l}
\end{array}\right)_{R} \stackrel{\oplus}{=}\left(\begin{array}{l}
\hat{\mathrm{o}}_{I n}^{+} \\
\underline{\hat{\mathrm{o}}}_{E l}
\end{array}\right)_{\underline{B}}=\left(\begin{array}{cc}
0 & \hat{\mathrm{o}}_{I n}^{+} \\
\hat{\mathrm{o}}_{E l} & 0
\end{array}\right) \stackrel{\oplus}{=}\left(\begin{array}{cc}
0 & \underline{\hat{\mathrm{o}}}_{I n}^{+} \\
\underline{\hat{\mathrm{o}}}_{E l} & 0
\end{array}\right)={ }_{1}\left(\begin{array}{c}
\hat{\mathrm{o}}_{I n}^{+}+\underline{\hat{\mathrm{o}}}_{I n}^{+} \\
\hat{\mathrm{o}}_{E l}+\underline{\hat{\mathrm{o}}}_{E l}
\end{array}\right)
$$

\subsection{The Quarkcolor}

The "massive coupling" builds the structure of couplings into quark (Figure $35)$.

We conjecture that the sub-IQuO components of a quark have the same direction of phase rotation, because so quark is electrically charged. This vouches

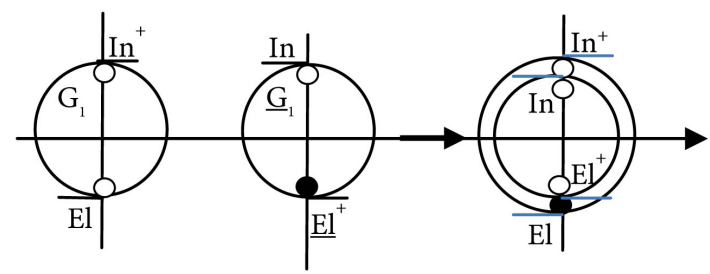

Figure 33. Gluon.

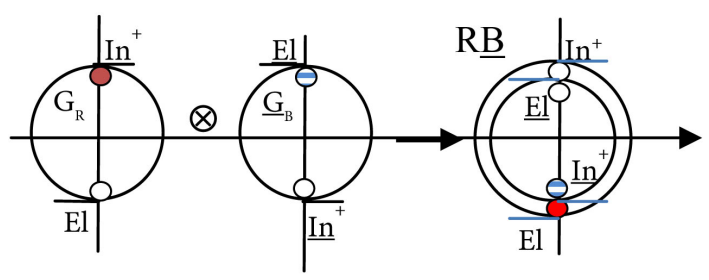

Figure 34. Rㅡ-gluon. 


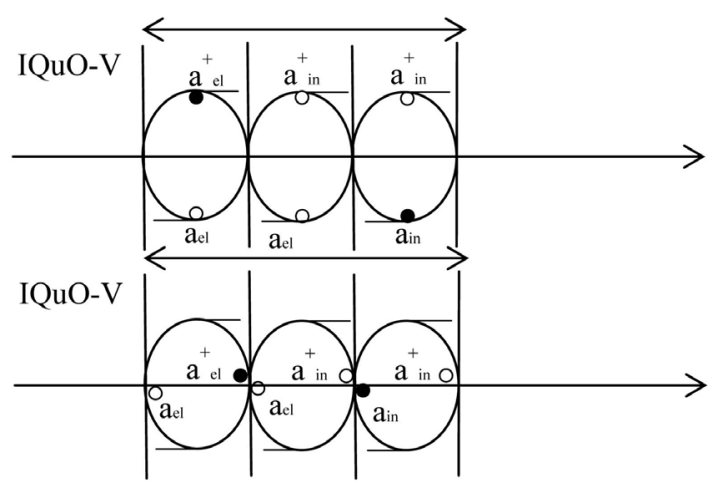

Next configuration

Figure 35. Configurations of internal Sub-IQuO into quark.

that flow direction of semi-quanta (isospin) inside the $\mathrm{IQuO}$ of the quark has not variations. Therefore, the configurations of an internal IQuO must be particular. Besides all IQuO of quark are quantum oscillators in eigenstate $\left(n=2^{*}\right)$ with 3 sub-oscillators. This determines that the third sub-oscillator is attacked to others two and this allows different possible IQuO configurations into a quark.

Nevertheless, the following configuration (Figure 36) of an IQuO-vertex:

is permissible but it is problematic because there can't be an exchange of energy between $\left(a_{e l}^{+}\right)_{1}$ and $\left(a_{e}\right)_{2}$ if they go in the same direction; in fact, $\left(\bullet_{e l}^{+}\right)$ is an operator of creation while $\left(o_{e l}\right)$ is instead annihilation operator. Idem it happens between $\left(a_{i n}^{+}\right)$and $\left(a_{i n}\right)$. Instead, this configuration is permissible (Figure 37):

This "blue-configuration" (see Figure 20) allows that a full semi-quantum (•) passes from sub-oscillator to another following: $\mathrm{A} \rightarrow \mathrm{B} \rightarrow \mathrm{C}$. The same it has happened in any another IQuO chain belonging to quark.

Even with others configurations, for having a blue quark it needs that inside of it there is always any sub-oscillator with blue color (see Figure 38).

We have so a quark with blue color $\left(\mathrm{Q}_{\mathrm{B}}\right)$. The index $(\mathrm{B})$ is associated to IQuO-0 $\left(a_{i n}, a_{e l}^{+}\right)$.

Nevertheless, this configuration is possible if we introduce into primary chain a gluon-chain having the blue color, i.e. a blue-antired gluon: $G_{B \underline{B}}$.

Besides, recall into quark the sides to be built between a superposition of IQuO (see Figure 27), therefore the configuration of chain-sides $\left(I_{B E}+I_{C H}\right)$ could be (Figure 39):

The S-IQuO is the representative IQuO of a colored Quark (in this case the quark is blue). The representation matrix of three sub-oscillators is:

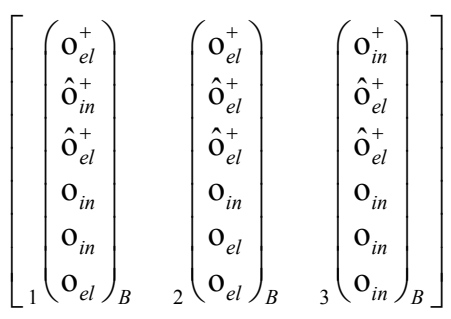




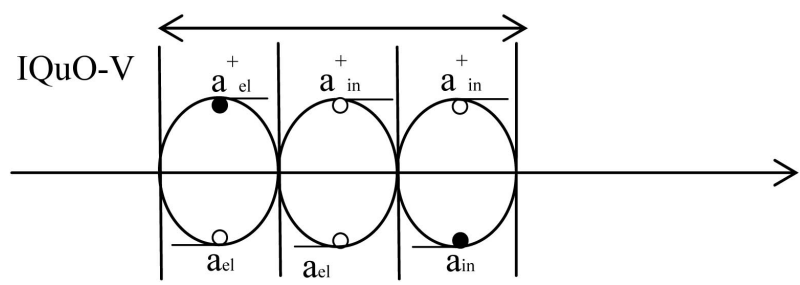

Figure 36. Possible configuration of IQuO vertex.

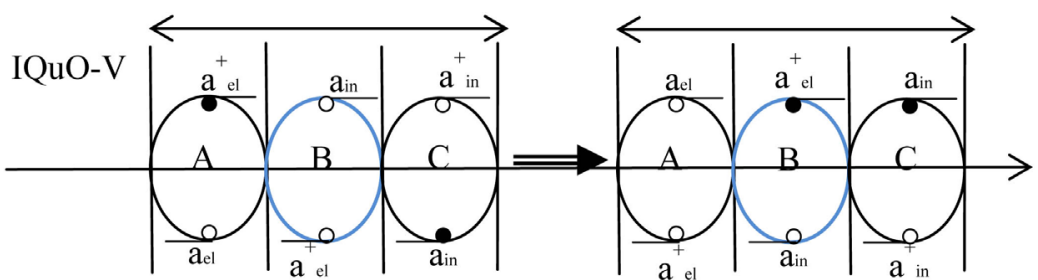

Figure 37. Permissible configuration of IQuO vertex.

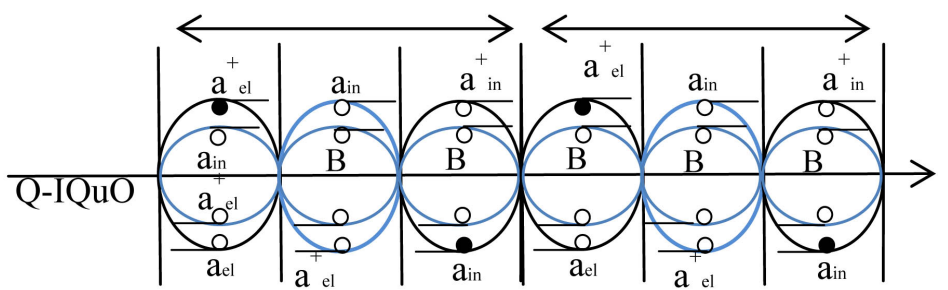

Figure 38. A chain of blue sub-oscillators inside a chain Q-IQuO.

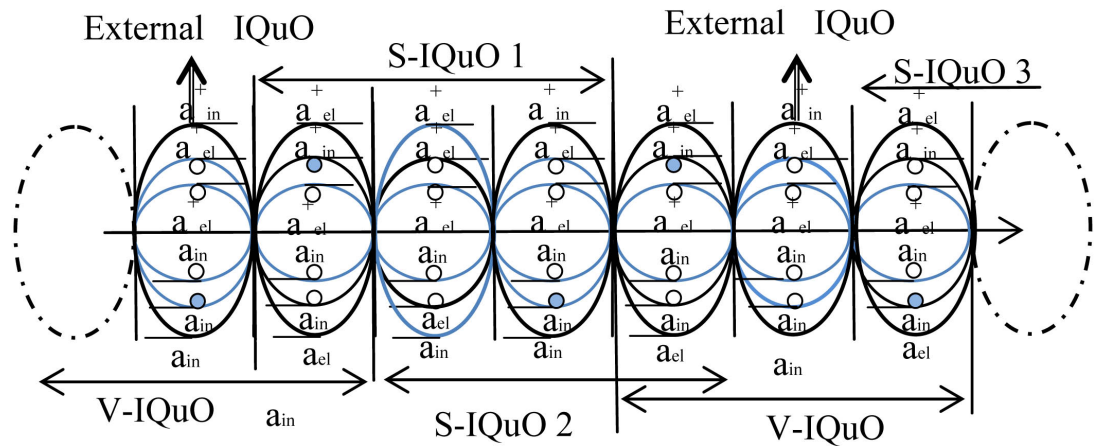

Figure 39. The chain-sides.

But the superposition between $\mathrm{I}_{\mathrm{CH}}$ and $\mathrm{I}_{\mathrm{BE}}$ in Figure 27 produces a phase shift into sub-oscillators. For realize the coupling between $\mathrm{I}_{\mathrm{CH}}$ and $\mathrm{I}_{\mathrm{BE}}$ and to conserve the isospin (the flow direction of semi-quanta inside to quark) it is need the existence of another internal gluon with blue color (B) and another with any anti-color $\left(\underline{G}_{i}\right)$. Therefore for realize the entanglement quantum between oscillator chains of a quark it need having two internal chains of gluons: $\left[G_{B \underline{R}}+G_{B \underline{R}}\right]$. In this way all sub-oscillators of sides and vertices of quark have the blue color (Figure 40).

We note that in all chains of quark it possible talk about internal superposition of sub-oscillators type gluons: in fact, note that the operator $\left(a_{i}, a_{j}^{+}\right)$over 


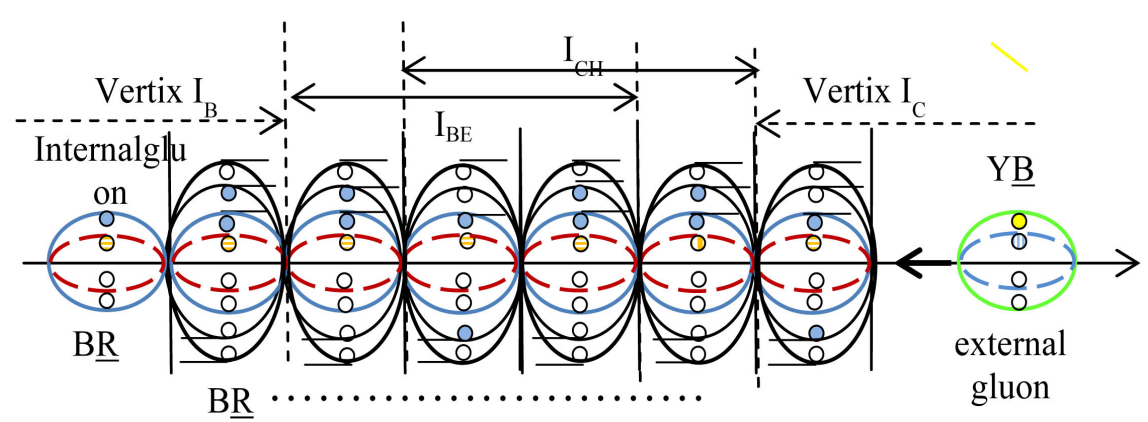

Figure 40. Structure at sub-oscillators into quark.

lap madding pairs of gluons $G_{i j}$ The matrices-operators of chain making the ( $\mathrm{I}_{\mathrm{BE}}$, $\mathrm{I}_{\mathrm{CH}}$ ) will indicate by $Q_{i p}$, while the internal ones by $G_{i j}$. We can admit only configurations allowing the passing of the full semi-quanta from sub-oscillator to next to obtain an isospinin variant. This shows that an isolated quark cannot change color, thank even to the internal gluons allowing that. Nevertheless, an isolated quark cannot exist and an only external gluon can change the color of quark. In this way, each sub-oscillator of quark is composed by one couple of operators: $\left(Q_{i p} G_{i k}\right)$. We have $\left.\left[Q_{i}\right]_{\text {quark }}=\left[\left(Q_{i j}\right)+\left(G_{i k}\right)\right] \equiv Q_{i j i \underline{i k}}\right)$.

In particular the matrix $\left[Q_{B}\right]=\left[\left(Q_{B_{2} R}\right)+\left(G_{B, \underline{Y}}\right)\right]_{B}$ will be:

$$
Q_{B}=Q_{(B R, B \underline{Y})}=\left[\begin{array}{ll}
\left(\begin{array}{l}
\left(\hat{\mathrm{o}}_{E l}^{+}\right. \\
\hat{\mathrm{o}}_{I n}
\end{array}\right)_{B} & \left.\left(\begin{array}{c}
\hat{\mathrm{o}}_{I n}^{+} \\
\hat{\mathrm{o}}_{E l}
\end{array}\right)_{R}\right)_{Q} \\
\left(\left(\begin{array}{cc}
\hat{\mathrm{o}}_{E l}^{+} \\
\hat{\mathrm{o}}_{I n}
\end{array}\right)_{B}\right. & \left.\left(\begin{array}{c}
\hat{\mathrm{o}}_{E l}^{+} \\
\underline{\hat{\mathrm{o}}}_{I n}
\end{array}\right)_{\underline{Y}}\right)_{G}
\end{array}\right]_{B}
$$

The coupling between quark and a gluon could change the color of quark, i.e. $Q_{B} \rightarrow Q_{R}$.

Then if $\left[Q_{B}\right]=\left[\left(Q_{B_{2} R}\right)+\left(G_{B, Y}\right)\right]_{B}$ we'll have $\left[Q_{B}+G_{R \underline{B}}\right]=Q_{R}$

That is:

$$
\begin{aligned}
{\left[Q_{B}+G_{R \underline{B}}\right] } & =\left[\left(Q_{B, R}\right)+\left(G_{B, \underline{Y}}\right)\right]+\left(G_{R \underline{B}}\right)=Q\left(_{B, R ; B, \underline{Y} ; R \underline{B}}\right) \\
& =Q\left(_{R B, R \underline{Y}}\right)=Q\left(_{R R, B \underline{Y}}\right)=Q_{R}
\end{aligned}
$$

In matrix representation:

$$
\begin{aligned}
& Q_{B}+G_{R \underline{B}}=Q_{(B R, B \underline{Y})}+G_{R \underline{B}}
\end{aligned}
$$

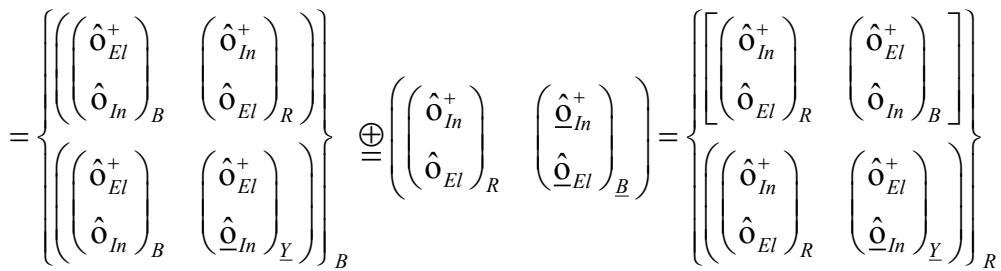

Another example:

$$
\left[Q_{B}+G_{Y \underline{B}}\right]=\left[\left(Q_{B, Y}\right)+\left(G_{B, \underline{R}}\right)\right]+\left(G_{Y \underline{B}}\right)=Q\left({ }_{Y B, Y \underline{R}}\right)=Q_{Y} .
$$




\section{Conclusions}

Talk about the physical properties as the electric charge, isospin, spin and color charge in some physics systems such as fields, it implies to add another freedom degree describing the corresponding phenomenon. We showed that all those properties can be connected to an internal freedom degree of the quantum oscillators representative of those fields. This shows that those systems are made of internal structures to associate with this internal freedom degree. Talking about internal structures in the system doesn't imply that it is composed by other parts linked or bounded together, but we could think instead that it is composed by coupled oscillators which establish a unique physical entity as an elementary particle. To introduce coupled oscillators system and geometrically structured we must think to peculiar quantum oscillators (IQuO) which internal freedom degree allows us to talk about those proprieties such as electric charge, isospin and color charge. The last one came from $\mathrm{IQuO}_{(\mathrm{n}=2)}$ and connected to the central sub-oscillator. In this way, the color charge states the existence of quantum oscillators represented by three sub-oscillators in which the energy is distributed with values of semi-quanta.

Even, note that the gluons are not only inside quark as its components but they are go-between two quarks for build the hadrons. Recall the mesons. Mesons are hadrons composed of a quark-antiquark pair. The elementary mesons are pions. We conjectured [3] [4] the IQuO structure of Aureus triangles built by couplings between $(\mathrm{u})$ and $(\mathrm{d})$ quarks. If we recall the proton structure (see Fig-

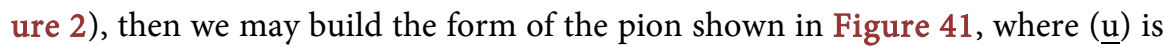
the u-antiquark.

The coupling between the $\underline{u}$-quark and d-quarks results with quadrangular structure.

To link the two ( $\underline{u}, d)$ quarks, it is necessary to add some junction IQuO between the sub-oscillators of the respective bases. The oscillators IQuO of the junction are the gluons. The "red" and "blue" colours of the sub-oscillators indicate the presence of descriptive coordinate: the "colour charge". The reciprocal phase shifts between G-IQuO and Q-IQuO of the quark structure must not dismantle the quark structure.

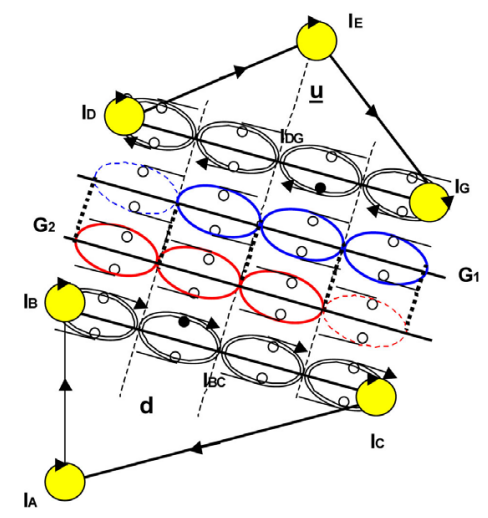

Figure 41. Pion structure. 
The figure also indicates the possibility of coupling between the IQuO of the quark chains, and between the G-IQuO.

\section{Conflicts of Interest}

The authors declare no conflicts of interest regarding the publication of this paper.

\section{References}

[1] Guido, G. (2012) The Substructure of a Quantum Oscillator Field. arXiv:1208.0948.

[2] Guido, G. (2014) The Substructure of a Quantum Field-Oscillator. Hadronic Journal, 37, 83 .

[3] Guido, G. (2017) About Structure of the Quarks. Hadronic Journal, 40, 22.

[4] Guido, G. (2017) Regarding the Structure of Quarks and Hadrons. Hadronic Journal, 40, 187-219.

[5] Khanna, M.P. (1999) Introduction To Particle Physics. Phi Learning Pvt. Ltd., Delhi.

[6] Letessier, J. and Rafelski, J. (2002) Hadrons and Quark-Gluon Plasma. Cambridge University Press, Cambridge, 30. https://doi.org/10.1017/CBO9780511534997

[7] Crawford Jr., F.S. (1965) Waves. McGraw-Hill, York.

[8] Sakurai, J.J. (1985) Modern Quantum Mechanics. Benjamin/Cummings Publishing Company Inc., San Francisco. 